\title{
POLYNOMIAL CONVOLUTIONS IN MAX-PLUS ALGEBRA
}

\author{
AMNON ROSENMANN, FRANZ LEHNER, AND ALJOŠA PEPERKO
}

\begin{abstract}
Recently, in a work that grew out of their exploration of interlacing polynomials, Marcus, Spielman and Srivastava [21] and Marcus [20] studied certain combinatorial polynomial convolutions. These convolutions preserve real-rootedness and capture expectations of characteristic polynomials of unitarily invariant random matrices, thus providing a link to free probability. We explore analogues of these types of convolutions in the setting of max-plus algebra. In this setting the max-permanent replaces the determinant and the maximum is the analogue of the expected value. Our results resemble those of Marcus et al., however, in contrast to the classical setting we obtain an exact and simple description of all roots.
\end{abstract}

\section{INTRODUCTION}

The study of polynomials, their roots and their critical points from the algebraic, analytic and geometric point of view has a long history (see e.g. the monographs [13, 23, 29], which emphasize the analyticgeometric approach). Recently, Marcus, Spielman and Srivastava [21] and Marcus [20] initiated the study of certain convolutions of polynomials that can implicitly be found in a paper by Walsh 33] from the early last century. They established a strong link to free probability by showing that these convolutions capture the expected characteristic polynomials of random matrices. However, their initial motivation

Date: February 22, 2018.

2010 Mathematics Subject Classification. Primary 15A80; Secondary 15A15, $26 \mathrm{C} 10$.

Key words and phrases. Max-plus algebra, max-convolution of maxpolynomials, Hadamard product, characteristic maxpolynomial.

Research of the first author was supported by the Austrian Science Fund (FWF) Projects P25510-N26 and P29355-N35. The third author was supported in part by a JESH grant of Austrian Academy of Sciences and he also acknowledges a partial support of the Slovenian Research Agency (grants P1-0222 and J1-8133). He thanks his colleagues and staff at TU Graz for their hospitality during his stay in Austria. 
came from the study of interlacing polynomials, which led to the solution of the Kadison-Singer problem [22], and they showed that these convolutions preserve the property of the roots being real numbers.

In the present paper we explore analogues of these types of convolution polynomials in the setting of max-plus algebra. In this setting the max-permanent replaces the determinant and the maximum is the analogue of the expected value. Our results resemble those of [21] in terms of the formulas (adjusted to the max-plus setting) for the convolution polynomials. In contrast to the classical setting, where only bounds on the maximal roots of the convolution polynomials are known, in max-plus algebra we obtain an exact and simple description of all the roots of the convolutions of maxpolynomials in terms of the roots of the involved maxpolynomials. Clearly, the preservation of the real-rootedness in the classical convolutions is irrelevant in max-plus algebra.

The paper is organized in the following way. In Section 2, after a brief introduction to max-plus algebra, we define a formal derivative of a formal maxpolynomial, which is utilized later for defining the max convolution. Then we discuss maxpolynomials in full canonical form [9], which are maxpolynomials that are formally fully-reduced into linear factors, or, equivalently, whose coefficients form a concave sequence (these polynomials are also called concavified polynomials or polynomials of maximum canonical form [2], maximally represented maxpolynomials 32] or least coefficient minpolynomials in the min-plus setting [15]).

We continue in Section 3 with a description of the different types of characteristic maxpolynomials that we deal with. In addition to the standard characteristic maxpolynomial we also consider the full characteristic maxpolynomial. It turns out that the latter is always in full canonical form and therefore more appropriate for the questions considered in the present paper than the former, which is in full canonical form only in special cases. As yet another variant we also introduce the Gram characteristic maxpolynomial of a matrix $A$, which is the characteristic maxpolynomial of the matrix $\left(A^{T} A\right)^{\circ \frac{1}{2}}$, i.e., the Hadamard root of $A^{T} A$, and whose roots are the maximal elements of the columns of $A$, which form the diagonal of $\left(A^{T} A\right)^{\circ \frac{1}{2}}$. As a function, the Gram characteristic maxpolynomial dominates the full characteristic maxpolynomial.

Next, we explore in Section 4 the convolution of characteristic maxpolynomials, which is the equivalent of the additive convolution of [21]. The additive convolution is defined over random orthogonal matrices, 
and this definition allows, when working with symmetric matrices, to reduce the computations to diagonal matrices, which makes life much easier. In the max-plus setting the set of orthogonal matrices consists only of permutation matrices, hence the computation of the maximum (the analogue of the expectation computation in the additive convolution) is over a finite set. In fact, also in the classical setting it suffices to perform the computations over the set of signed permutation matrices (but, on the other hand, also over the set of unitary matrices).

In max-plus algebra we cannot achieve diagonalization due to the fact that there are no "negative" elements with respect to the max operation. It turns out, however, that the set of "principally dominant" matrices, the matrices whose characteristic maxpolynomial equals the full characteristic maxpolynomial, suffices for showing that the convolution of the characteristic maxpolynomials of matrices $A$ and $B$ equals the maximum of the characteristic maxpolynomials of the max operation between $A$ and a conjugate of $B$, where the maximum is taken over the set of permutation matrices (Theorem 4.10).

When the matrices are not principally dominant then Theorem 4.10 does not hold even when the matrices are symmetric, as shown in Example 4.11. However, when we allow the permutations to operate on rows and columns of $B$ independently then a max convolution theorem similar to Theorem 4.10 does hold. In this case the computation is executed with respect to the full characteristic maxpolynomial (Theorem 4.5).

As for the roots of the convolution maxpolynomial, we obtain a formula which is similar to the classical case: the maximum (as opposed to expectation) is over a set of maxpolynomials, where each root of these maxpolynomials is expressed as the maximum (as opposed to sum) of a root of the full characteristic maxpolynomial of $A$ and a root of the full characteristic maxpolynomial of $B$. However, in the max-plus case the result is that the $n$ roots of the max convolution are exactly the $n$ maximal roots among those of the involved characteristic maxpolynomials, whereas in the standard additive convolution only a bound on the maximal root of the convolution polynomial can be given [33].

We close Section 4 with the "max-row convolution", which is the analogue of the "asymmetric additive convolution" of [21]. The maxrow convolution is defined over the characteristic maxpolynomials of $\left(A A^{T}\right)^{\circ \frac{1}{2}}$ and $\left(B B^{T}\right)^{\circ \frac{1}{2}}$ and its roots are the maximal roots among the set obtained by picking the maximal element in each row of $A$ and each row of $B$. 
In the final section, after discussing properties of Hadamard products (element-wise products) of maxpolynomials and of matrices, we characterize a convolution which relates the Hadamard products of matrices and maxpolynomials to each other. In Theorem 5.5 we show that the maximum over the full characteristic maxpolynomials of the Hadamard products of the matrix $A$ and permutations of the matrix $B$ equals the Hadamard product of the full characteristic maxpolynomials of $A$ and $B$. The ordered list of the roots of the resulting maxpolynomial consists of the product (sum in standard arithmetic) of the ordered lists of the roots of the operands.

The plus convolution, which is the analogue of the product convolution in classical mathematics, is more specialized than the max convolution in the sense that the rows of $A$ and the columns of $B$ should have a common partition with respect to the positions of the maximal elements. In this case, we show in Theorem 5.6 that the Hadamard product of the characteristic maxpolynomials of $\left(A A^{T}\right)^{\circ \frac{1}{2}}$ and $\left(B B^{T}\right)^{\circ \frac{1}{2}}$ equals the maximum (over permutation matrices) of the characteristic maxpolynomials of the product of $A$ and a permutation of $B$.

As for the roots of the plus convolution maxpolynomial, we obtain a formula which is similar to the classical case: the maximum (as opposed to expectation) is over a set of maxpolynomials, where each root of these maxpolynomials is expressed as the product of a root of the characteristic maxpolynomial of $\left(A A^{T}\right)^{\circ \frac{1}{2}}$ and a root of the characteristic maxpolynomial of $\left(B B^{T}\right)^{\circ \frac{1}{2}}$. Again, the ordered list of the roots of the resulting maxpolynomial consists of the product (sum in standard arithmetic) of the ordered lists of the roots of the operands, whereas in the standard multiplicative convolution only a bound on the maximal root of the convolution polynomial is given [31.

\section{MAX-PLUS ALGEBRA}

In its current setting, max-plus algebra is a relatively new field, which emerged from several branches of mathematics simultaneously. It is an algebra over the ordered, idempotent semiring (in fact, semifield) $\mathbb{R}_{\max }=\mathbb{R} \cup\{-\infty\}$, equipped with the operations of addition $a \oplus b=\max (a, b)$ and multiplication $a \odot b=a+b$, with the unit elements $\varepsilon=-\infty$ (for addition) and $\mathbb{1}=0$ (for multiplication). As in standard arithmetic, the operations of addition and multiplication are associative and commutative, and multiplication is distributive over addition. Matrix and polynomial operations are defined similarly to 
their standard counterparts, with the max-plus operations replacing the standard operations.

Max-plus algebra is isomorphic to min-plus algebra (also known as tropical algebra), which is the semifield $\mathbb{R}_{\text {min }}=\mathbb{R} \cup\{\infty\}$, where addition is replaced by minimum and multiplication by addition, and also to max-times algebra $\mathbb{R}_{+}$, where addition is replaced by maximum and multiplication is the same as in standard arithmetic. For more on max-plus algebra we refer to the monograph of Butkovič [6]. Max-plus algebra is a part of a broader branch of mathematics, "idempotent mathematics", which was developed mainly by Maslov and his collaborators (see [18] for a brief introduction).

Max-plus algebra, together with its isomorphic versions, provides an attractive way of describing a class of non-linear problems appearing for instance in manufacturing and transportation scheduling, information technology, discrete event-dynamic systems, combinatorial optimization, mathematical physics and DNA analysis (see e.g. [6, 2, 26, 19, 18, 3, 25, 7], and the references cited there).

For the sake of readability, we mostly suppress the multiplication sign $\odot$, writing $a b$ instead of $a \odot b$ and $a x^{3}$ instead of $a \odot x^{\odot 3}$ or $a \odot x \odot x \odot x$. Also, when an indeterminate $x$ appears without a coefficient, as in $x^{n}$, then its coefficient is naturally the multiplicative identity element, i.e. 0 .

\subsection{Maxpolynomials and tropical roots. A (formal) maxpoly- nomial is an expression of the form}

$$
p(x)=\bigoplus_{k=0}^{d} a_{k} x^{k}=\max \left\{a_{k}+k x: k=0,1, \ldots, d\right\},
$$

where $a_{0}, \ldots, a_{d} \in \mathbb{R}_{\max }$ and $x$ is a formal indeterminate. We assume that $a_{d} \neq \varepsilon$ and then $p(x)$ is of degree $d$, unless $p(x)=\varepsilon x^{0}$, the null maxpolynomial, which is of degree $-\infty$. The terms $a_{k} x^{k}$ are called the monomials constituting $p(x)$ and normally the monomial $a_{k} x^{k}$ is omitted when $a_{k}=\varepsilon$. The set of maxpolynomials form a semiring (there is no additive inverse) $\mathbb{R}_{\max }[x]$ with respect to the max-plus operations of addition and multiplication. Each expression in $\mathbb{R}_{\max }[x]$ that is the result of application of these max-plus operations to maxpolynomials can be reduced to a unique canonical form as in (2.1) according to the rules of $\mathbb{R}_{\max }$. Hence, we say that two maxpolynomials are (formally) equal if they have the same canonical form.

A maxpolynomial $p(x)$ induces a convex, piecewise-affine function $\hat{p}(x)$ on $\mathbb{R}_{\max }$. Unlike the situation in standard arithmetic, two distinct 
formal maxpolynomials $p_{1}(x)$ and $p_{2}(x)$ may represent the same polynomial function, that is, $\hat{p}_{1}(x)=\hat{p}_{2}(x)$ as functions. The (max-plus or tropical) roots of a maxpolynomial $p(x)$ are the points at which $\hat{p}(x)$ is non-differentiable. The multiplicity of a root equals the change of the slope of $\hat{p}(x)$ at that root. Equivalently, the roots of $p(x)$ are the values $r \neq \varepsilon$ of $x$ at which several monomials $a_{k} x^{k}, a_{k+1} x^{k+1}, \ldots, a_{k+l} x^{k+l}$ in the canonical form satisfy $a_{k} r^{k}=a_{k+1} r^{k+1}=\cdots=a_{k+l} r^{k+l}=\hat{p}(r)$ and the multiplicity of the root $r$ is then $l$, the difference between the largest and the smallest index of these monomials. We also count $\varepsilon=-\infty$ as a root with multiplicity $l$ whenever $a_{0}, a_{1}, \ldots, a_{l-1}$ are all equal to $\varepsilon$ and $a_{l} \neq \varepsilon$. In this case the monomials $\varepsilon x^{i}, 0 \leq i \leq l-1$, represent the constant function $\varepsilon$, which intersects the line $\hat{p}_{k}(x)=a_{k} x^{k}$ at $x=\varepsilon$.

\subsection{Derivatives of maxpolynomials.}

Definition 2.1. Given a maxpolynomial $p(x) \in \mathbb{R}_{\max }[x]$, we define its max-plus derivative to be the result of applying the max-linear shift operator (or "annihilation operator") $\partial_{x}: \mathbb{R}_{\max }[x] \rightarrow \mathbb{R}_{\max }[x]$ defined by

$$
\partial_{x} x^{k}= \begin{cases}x^{k-1} & k \geq 1 \\ \varepsilon & k=0\end{cases}
$$

i.e.,

$$
\partial_{x}\left(\bigoplus_{i=0}^{n} a_{i} x^{i}\right)=\bigoplus_{i=0}^{n-1} a_{i+1} x^{i} .
$$

We will use the notation $p^{\prime}(x)$ for $\partial_{x} p(x)$ and $p^{(i)}(x)$ for $\partial_{x}^{i} p(x)$ wherever convenient. This is a derivation (Proposition 2.2 below); in fact, it is the unique derivation on $\mathbb{R}_{\max }[x]$ satisfying

(i) $a^{\prime}=\varepsilon$ for every $a \in \mathbb{R}_{\max }$,

(ii) $x^{\prime}=0$,

i.e., the unique operator on $\mathbb{R}_{\max }[x]$ satisfying

(iii) $(p \oplus q)^{\prime}(x)=p^{\prime}(x) \oplus q^{\prime}(x)$ (linearity),

(iv) $(p q)^{\prime}(x)=\left(p^{\prime} q\right)(x) \oplus\left(p q^{\prime}\right)(x)$ (Leibniz's rule), for every $p(x), q(x) \in \mathbb{R}_{\max }[x]$.

One then shows (e.g., by induction) that the following iterated Leibniz's rule holds:

Proposition 2.2 (Leibniz's rule). Let $p(x), q(x) \in \mathbb{R}_{\max }[x]$. Then

$$
(p q)^{(k)}(x)=\bigoplus_{i=0}^{k} p^{(i)}(x) q^{(k-i)}(x) \text {. }
$$


Remark 2.3. 1. For another form of derivative in tropical mathematics we refer to the "layered derivative", which is defined in 17.

2. We emphasize that our derivation operates on a purely formal level. The derivatives of different maxpolynomial $p(x)$ representatives of a piecewise-linear convex function $\hat{p}(x)$ need not coincide, e.g., the maxpolynomials $p(x)=x^{2} \oplus x \oplus 0$ and $q(x)=x^{2} \oplus 0$ are functionally equivalent: $\hat{p}(x)=\hat{q}(x)$, however, the derivatives $p^{\prime}(x)=x \oplus 0$ and $q^{\prime}(x)=x$ are not.

Proposition 2.7 below says that roughly speaking, the derivative of a maxpolynomial function can be defined as the operation of removing the smallest root (and, in general, a possible reduction in the value of other smallest roots).

3. Given a maxpolynomial $p(x)=\bigoplus_{i=0}^{n} a_{i} x^{i}$, it can be represented via its Taylor expansion around $\varepsilon$ as

$$
\bigoplus_{i=0}^{n} \widehat{p^{(i)}}(\varepsilon) x^{i}
$$

however, the Taylor expansion does not hold in other points.

\subsection{Full canonical maxpolynomials.}

Definition 2.4. A maxpolynomial $p(x)=\bigoplus_{i=0}^{n} a_{i} x^{i}$ is in full canonical form [9] (or is an FCF maxpolynomial) if it is either constant or can be decomposed into linear factors: $p(x)=a_{n}\left(x \oplus s_{1}\right) \cdots\left(x \oplus s_{n}\right)$.

As before, we adopt the convention that monomials with coefficient $\varepsilon$ are not written out and then it is clear that every monomial is in full canonical form; indeed $a x^{n}=a(x \oplus \varepsilon)^{n}$.

If we partition the set of maxpolynomials in $\mathbb{R}_{\max }[x]$ according to their functional property then each equivalence class has a unique FCF maxpolynomial that represents it. For example, $p(x)=x^{4} \oplus x^{3} \oplus x^{2}=$ $(x \oplus 0)^{2}(x \oplus \varepsilon)^{2}$ is the full canonical representative of the equivalence class of all maxpolynomials $q(x)$ with $\hat{q}(x)=\hat{p}(x)$ which contains, among others, the maxpolynomials $x^{4} \oplus x^{2}$ and $x^{4} \oplus(-1) x^{3} \oplus x^{2}$ which are not FCF.

In the following proposition we adopt the convention $-\infty-(-\infty)=$ $-\infty$.

Proposition 2.5. Let $p(x)=\bigoplus_{i=0}^{n} a_{i} x^{i}$ and let $r_{1} \leq r_{2} \leq \cdots \leq r_{n}$ be its roots. Then the following are equivalent.

(i) $p(x)$ is in full canonical form: $p(x)=a_{n}\left(x \oplus s_{1}\right) \cdots\left(x \oplus s_{n}\right)$ for some $s_{1}, \ldots, s_{n}$.

(ii) $p(x)=a_{n}\left(x \oplus r_{1}\right) \cdots\left(x \oplus r_{n}\right)$. 
(iii) $r_{i}=a_{i-1}-a_{i}, i=1, \ldots, n$.

(iv) Concavity: $a_{i-1}-a_{i} \leq a_{i}-a_{i+1}, i=1, \ldots, n-1$.

(v) $\hat{p}\left(r_{i}\right)=a_{i} r_{i}^{i}\left(a_{i}+i r_{i}\right.$ in standard arithmetic) for $i=1, \ldots, n$.

Proof. The equivalences follow from the definition of the tropical roots of a maxpolynomial and from simple manipulations of the above expressions.

We demonstrate some of the implications. Suppose that $(i)$ holds and assume that $s_{1} \leq \cdots \leq s_{n}$. Expanding the expression $a_{n}\left(x \oplus s_{1}\right) \cdots(x \oplus$ $\left.s_{n}\right)$ gives: $a_{i}=a_{n}+s_{i+1}+\cdots+s_{n}$ for $i=0, \ldots, n-1$. It follows that $a_{i-k}-a_{i}=\left(s_{i-k+1}+\cdots+s_{n}\right)-\left(s_{i+1}+\cdots+s_{n}\right)=s_{i-k+1}+\cdots+s_{i}$ for $k \geq 1$. Then $\left(a_{i-k}-a_{i}\right) / k \leq k s_{i} / k=s_{i}$ and thus $r_{i}=s_{i}=a_{i-1}-a_{i}$.

As for $(v)$, geometrically, at each root $x=r_{i}$ of $p(x)$, the convex piecewise-linear function $\hat{p}(x)$ has a corner at the point $\left(r_{i}, \hat{p}\left(r_{i}\right)\right)(\hat{p}(x)$ is non-differentiable at $\left.x=r_{i}\right)$, and when $p(x)$ is in full canonical form then when $r_{i}=r_{i+1}=\cdots=r_{i+l}$ all lines $a_{i+j} x^{i+j}, j=0, \ldots, l$, pass through this corner.

The sum of maxpolynomials in full canonical form need not be in full canonical form; however the next proposition shows that FCF polynomials are closed under the operation of derivative and multiplication.

Proposition 2.6. Let $p(x), q(x)$ be FCF maxpolynomials. Then

(i) $p^{\prime}(x)$ is $F C F$.

(ii) $(p q)(x)$ is FCF.

Proof. Let us assume that $p(x)=\bigoplus_{i=0}^{n} a_{i} x^{i}$ and $q(x)=\bigoplus_{i=0}^{m} b_{i} x^{i}$ are not constant, otherwise the proof follows immediately. Since $p(x)$ and $q(x)$ are FCF maxpolynomials they can be linearly factored: $p(x)=$ $a_{n}\left(x \oplus r_{1}\right) \cdots\left(x \oplus r_{n}\right), q(x)=b_{m}\left(x \oplus s_{1}\right) \cdots\left(x \oplus s_{m}\right)$. The product of the maxpolynomials is then $(p q)(x)=a_{n} b_{m}\left(x \oplus r_{1}\right) \cdots\left(x \oplus r_{n}\right)(x \oplus$ $\left.s_{1}\right) \cdots\left(x \oplus s_{m}\right)$, which is also a product of linear factors and hence FCF.

The derivative of $p(x)$ is $p^{\prime}(x)=\bigoplus_{i=1}^{n} a_{i} x^{i-1}$ and the differences $a_{i-1}-a_{i}$ (that satisfy the concavity condition) remain in $f^{\prime}(x)$, except for $r_{1}=a_{0}-a_{1}$. It follows that $p^{\prime}(x)=a_{n}\left(x \oplus r_{2}\right) \cdots\left(x \oplus r_{n}\right)$, which is again in full canonical form.

The next proposition shows that the derivative acts on FCF maxpolynomials as a shift on the roots as well.

Proposition 2.7. Let $p(x)$ be an FCF maxpolynomial with roots $r_{1} \leq$ $r_{2} \leq \cdots \leq r_{n}$. Then the roots of $p^{\prime}(x)$ are $r_{2} \leq r_{3} \leq \cdots \leq r_{n}$. 
Proof. The result follows from the proof of Proposition 2.6. Alternatively, by applying Leibniz's rule to the factorization

$$
p(x)=a_{n}\left(x \oplus r_{1}\right)\left(x \oplus r_{2}\right) \cdots\left(x \oplus r_{n}\right)
$$

we obtain

$$
p^{\prime}(x)=a_{n} \bigoplus_{k=1}^{n}\left(x \oplus r_{1}\right) \cdots\left(x \oplus r_{k-1}\right)\left(x \oplus r_{k+1}\right) \cdots\left(x \oplus r_{n}\right),
$$

that is, the term $\left(x \oplus r_{k}\right)$ is eliminated in the $k$-th summand. Now it is clear that the maximal value is attained when the smallest root is eliminated.

Remark 2.8. Iterating Proposition 2.7 establishes the following characterization of FCF maxpolynomials: $p(x)$ is in full canonical form if and only if the roots of $p^{(k)}(x)$ are the largest $n-k$ roots of $p(x)$.

Example 2.9. Let $p(x)=(-1) x^{2} \oplus x \oplus 1=(-1)(x \oplus 1)^{2}$ and $q(x)=$ $x^{2} \oplus x \oplus 0=(x \oplus 0)^{2}$. Then $p^{\prime}(x)=(-1) x \oplus 0=(-1)(x \oplus 1)$ and $p q(x)=(-1) x^{4} \oplus x^{3} \oplus 1 x^{2} \oplus 1 x \oplus 1=(-1)(x \oplus 0)^{2}(x \oplus 1)^{2}$. Hence, $p(x), q(x), p^{\prime}(x)$ and $(p q)(x)$ can be decomposed into linear factors and thus are FCF maxpolynomials.

Here $(p \oplus q)(x)$ is not in full canonical form: $(p \oplus q)(x)=x^{2} \oplus x \oplus 1$ has roots $r_{1}=r_{2}=\frac{1}{2}$. However, $\left(x \oplus \frac{1}{2}\right)^{2}=x^{2} \oplus \frac{1}{2} x \oplus 1 \neq(p \oplus q)(x)$ as a maxpolynomial expression.

\section{The CHARACTERISTIC MAXPOLYNOMIAlS OF A MATRIX}

Definition 3.1. Let $A \in \mathbb{R}_{\max }^{n \times n}$ be an $n \times n$ matrix over $\mathbb{R}_{\max }$. The max-plus permanent of $A$ is

$$
\operatorname{perm}(A)=\bigoplus_{\sigma \in \mathfrak{S}_{n}} a_{1 \sigma(1)} \cdots a_{n \sigma(n)},
$$

where $\mathfrak{S}_{n}$ is the group of permutations on $[n]=\{1, \ldots, n\}$. For subsets $I, J \subseteq[n]$ of equal cardinality the permanent of the submatrix $A_{I, J}=$ $\left[a_{i, j}\right]_{i \in I, j \in J}$ is called the $(I, J)$-minor of $A$. The principal minors are the minors corresponding to $I=J$.

Definition 3.2. The characteristic maxpolynomial of $A$, defined in [8], is

$$
\chi_{A}(x)=\operatorname{perm}(x I \oplus A),
$$

where $I$ is the max-plus identity matrix with all entries on the main diagonal being 0 and all off-diagonal entries being $\varepsilon$. This is a polynomial of degree $n$, say $\chi_{A}(x)=\bigoplus_{k=0}^{n} c_{k} x^{k}$, with $c_{k}=\delta_{n-k}(A)$, 
$k=0, \ldots, n-1$, where

$$
\begin{aligned}
\delta_{k}(A) & =\bigoplus_{\substack{I \subseteq[n] \\
|I|=k}} \operatorname{perm}\left(A_{I, I}\right) \\
& =\bigoplus_{i_{1}, i_{2}, \ldots, i_{k}} \bigoplus_{\sigma \in \mathfrak{S}_{k}} a_{i_{1} i_{\sigma(1)}} a_{i_{2} i_{\sigma(2)}} \cdots a_{i_{k} i_{\sigma(k)}}
\end{aligned}
$$

is the maximal value of the principal minors of order $k$ of $A$.

As shown in [1, the (tropical) roots of the characteristic maxpolynomial $\chi_{A}(x)$, which are called the max-plus eigenvalues of $A$, can be asymptotically computed from the eigenvalues of an associated parametrized classical matrix with exponential entries.

Definition 3.3. We define the full characteristic maxpolynomial of $A$ to be

$$
\bar{\chi}_{A}(x)=\operatorname{perm}(x 0 \oplus A),
$$

where 0 is the matrix with zeroes in all its entries. The difference between $\bar{\chi}_{A}(x)$ and $\chi_{A}(x)$ is that in $\bar{\chi}_{A}(x)$ the indeterminate $x$ appears in all entries instead of just on the diagonal. It follows that

$$
\bar{\chi}_{A}(x) \geq \chi_{A}(x)
$$

as maxpolynomial functions.

Again, this is a polynomial of degree $n$, say $\bar{\chi}_{A}(x)=\bigoplus_{k=0}^{n} d_{k} x^{k}$, whose $k$-th coefficient $d_{k}=\eta_{n-k}(A), k=0, \ldots, n-1$, is the maximal value of all minors of order $n-k$ of $A$, that is,

$$
\begin{aligned}
\eta_{k}(A) & =\bigoplus_{\substack{I, J \subseteq[n] \\
|I|=|J|=k \\
|I|=k}} \operatorname{perm}\left(A_{I, J}\right) \\
& =\bigoplus_{\substack{i_{1}, i_{2}, \ldots, i_{k} \\
j_{1}, j_{2}, \ldots, j_{k}}} a_{i_{1} j_{1}} a_{i_{2} j_{2}} \cdots a_{i_{k} j_{k}}
\end{aligned}
$$

where the last sum in (3.2) runs over all pairs of ordered $k$-tuples of distinct indices. The relation to the principal minors is

$$
\delta_{k}(A) \leq \eta_{k}(A)=\bigoplus_{P} \delta_{k}(A P)
$$

where the last sum runs over all permutation matrices.

Similarly to eigenvalues, the (tropical) roots of the full characteristic maxpolynomial $\bar{\chi}_{A}(x)$, which are called the max-plus singular values of $A$, can be asymptotically computed from the singular values of an 
associated parametrized classical matrix with exponential entries (see [16] and [11]).

The characteristic maxpolynomial is not necessarily in full canonical form. However, this is the case for the full characteristic maxpolynomial, which makes it a better choice for the investigation of convolutions of maxpolynomials in following sections.

Theorem 3.4. The full characteristic maxpolynomial is FCF.

Proof. Let $\bar{\chi}_{A}(x)=\bigoplus_{k=0}^{n} d_{k} x^{k}$ with $d_{n}=0$, be the full characteristic maxpolynomial of the $n \times n$ matrix $A$. The $(n-k)$ th coefficient of $\bar{\chi}_{A}(x), d_{n-k}=\eta_{k}, k=1, \ldots, n$, equals the maximal permanent over all submatrices of $A$ of order $k$, which is the optimal value of the corresponding $k$-cardinality assignment problem [5] and the values $\eta_{k}$ form a concave sequence, that is, the inequalities $\eta_{k+1}-\eta_{k} \geq \eta_{k+2}-\eta_{k+1}$, $k=0, \ldots, n-2$ hold. By Proposition 2.5 the full characteristic maxpolynomial $\bar{\chi}_{A}(x)$ is in full canonical form.

Indeed, the $k$-cardinality assignment problem can be formulated as an integer programming (IP) problem over the variables $x_{i, j}, 1 \leq i, j \leq$ $n$ as follows [12]:

$$
\sum_{i=1}^{n} \sum_{j=1}^{n} a_{i j} x_{i j} \rightarrow \max !
$$

subject to the conditions

$$
\begin{aligned}
& \sum_{j=1}^{n} x_{i j} \leq 1 \quad(i=1, \ldots, n), \\
& \sum_{i=1}^{n} x_{i j} \leq 1 \quad(j=1, \ldots, n), \\
& \sum_{i=1}^{n} \sum_{j=1}^{n} x_{i j}=k, \\
& x_{i j} \in\{0,1\} \quad(i=1, \ldots, n, \quad j=1, \ldots, n) .
\end{aligned}
$$

If we replace the discrete condition (3.3d) by the linear condition

$$
\text { (3.3d) } \quad x_{i j} \geq 0 \quad(i=1, \ldots, n, \quad j=1, \ldots, n) \text {, }
$$

we obtain the corresponding relaxed linear programming (LP) problem.

In general, there is no reason for the solution of the (LP) to be integer valued and to solve the underlying (IP) problem. This is however the case if the system matrix is totally unimodular, see [24, Lemma 8.2.4] or [30, Chapter 19]. The $k$-assignment problem satisfies this condition [12, Theorem 1]. It is a well known fact in the theory of linear programming 
that the solution of the LP minimization problem is a convex function of the constraint vector [4, Theorem 5.1] and it follows that the sequence $\eta_{k}$ of solutions of the our max problem is concave.

It is also beneficial to obtain the same geometric description of the solution set by representing the assignment problem in the language of a network flow problem [10].

3.1. The Gram characteristic maxpolynomial. We start with a few definitions.

Definition 3.5. Given an $n \times n$ matrix $A \in \mathbb{R}_{\max }^{n \times n}$ we call $G=A^{T} A$ the max-plus Gram matrix of $A$ and $\operatorname{perm}(G)$ the max-plus Gram permanent of $A$.

Given two (column) vectors $u, v$ of size $n$, let $\langle u, v\rangle=u^{T} v$ be their max-plus scalar product. The max-plus norm of $v$ is $\|v\|=\langle v, v\rangle^{\frac{1}{2}}$, the maximal element of $v$. Then the max-plus version of the CauchyBunyakovsky-Schwarz inequality holds:

$$
\langle u, v\rangle \leq\|u\|\|v\|
$$

with equality if and only if the maxima of $u$ and $v$ occur at the same index, i.e., if there is $1 \leq i \leq n$ such that $\|u\|=u_{i}$ and $\|v\|=v_{i}$.

In the following we denote by $a_{i}$ the column vectors of $A$.

Proposition 3.6. Let $A=\left(a_{i j}\right) \in \mathbb{R}_{\max }^{n \times n}$ and let $G=\left(g_{i j}\right)=A^{T} A$ be its Gram matrix. Then the Gram permanent of $A$ is

$$
\operatorname{perm}(G)=\left\|a_{1}\right\|^{2}\left\|a_{2}\right\|^{2} \cdots\left\|a_{n}\right\|^{2}=g_{11} g_{22} \cdots g_{n n} .
$$

Proof. The entries of the matrix $G$ are

$$
g_{i j}=\left\langle a_{i}, a_{j}\right\rangle
$$

and

$$
\operatorname{perm}(G)=\bigoplus_{\pi \in \mathfrak{S}_{n}}\left\langle a_{1}, a_{\pi(1)}\right\rangle\left\langle a_{2}, a_{\pi(2)}\right\rangle \cdots\left\langle a_{n}, a_{\pi(n)}\right\rangle .
$$

Now, for a fixed permutation $\pi$ we apply the inequality (3.4) and obtain

$$
\begin{aligned}
\left\langle a_{1}, a_{\pi(1)}\right\rangle\left\langle a_{2}, a_{\pi(2)}\right\rangle \cdots\left\langle a_{n}, a_{\pi(n)}\right\rangle & \leq\left\|a_{1}\right\|\left\|a_{\pi(1)}\right\|\left\|a_{2}\right\|\left\|a_{\pi(2)}\right\| \cdots\left\|a_{n}\right\|\left\|a_{\pi(n)}\right\| \\
& =\left\|a_{1}\right\|^{2}\left\|a_{2}\right\|^{2} \cdots\left\|a_{n}\right\|^{2} \\
& =g_{11} g_{22} \cdots g_{n n},
\end{aligned}
$$

i.e., the maximal value is attained for $\pi=1$. 
Given a matrix $M$, we denote by $M^{\circ \frac{1}{2}}$ the Hadamard root of $M$, i.e., the result of multiplying (in standard arithmetic) $M$ by the scalar $\frac{1}{2}$. For more on Hadamard product and Hadamard power of matrices, see Subsection 5.2 .

Definition 3.7. We denote by $\widehat{A}$ the Hadamard root of the Gram matrix of $A$,

$$
\widehat{A}=G^{\circ \frac{1}{2}}=\left(A^{T} A\right)^{\circ \frac{1}{2}}
$$

and call $\chi_{\widehat{A}}(x)$ the Gram characteristic maxpolynomial of $A$.

The same arguments as in the proof of Proposition 3.6 together with (3.1) Show that we only need to consider the diagonal elements of $\widehat{A}$, that is, $\chi_{\widehat{A}}(x)$ is the FCF maxpolynomial

$$
\begin{aligned}
\chi_{\widehat{A}}(x) & =\left(x \oplus \widehat{A}_{11}\right)\left(x \oplus \widehat{A}_{22}\right) \cdots\left(x \oplus \widehat{A}_{n n}\right) \\
& =\left(x \oplus m_{1}\right)\left(x \oplus m_{2}\right) \cdots\left(x \oplus m_{n}\right),
\end{aligned}
$$

where $m_{i}=\left\|a_{i}\right\|$ is the maximal element of column $i$ of $A$. If follows that

$$
\chi_{\widehat{A}}(x) \geq \bar{\chi}_{A}(x)
$$

as maxpolynomial functions.

\section{MAX CONVOLUTION}

\subsection{Max convolution of maxpolynomials.}

Definition 4.1. Given two maxpolynomials $p(x), q(x)$, their max convolution of order $k$ (or $k$-th max convolution) is the $k$-th derivative of their max-plus product:

$$
\left(p \boxplus_{k} q\right)(x)=(p q)^{(k)}(x)=\bigoplus_{i=0}^{k} p^{(i)}(x) q^{(k-i)}(x) .
$$

For example, if $q(x)=0$, the zero maxpolynomial, then $\left(p \boxplus_{k} 0\right)(x)$ is the $k$-th derivative of $p(x)$, and if $k=0$ then $\left(p \boxplus_{0} q\right)(x)$ is the product of $p(x)$ and $q(x)$.

Proposition 4.2. For any FCF maxpolynomials $p(x), q(x)$ of degree $m, n$ respectively the following holds.

(i) For every $k<m+n$ the max-convolution polynomial $\left(p \boxplus_{k} q\right)(x)$ is an FCF maxpolynomial of degree $m+n-k$.

(ii) The roots of $\left(p \boxplus_{k} q\right)(x)$ are the maximal $m+n-k$ roots (including multiplicities) among the roots of $p(x)$ and the roots of $q(x)$. 
(iii) In particular, when $p(x)=\bigodot_{i=1}^{n}\left(x \oplus r_{i}\right)$ and $q(x)=\bigodot_{i=1}^{n}\left(x \oplus s_{i}\right)$ then

$$
\left(p \boxplus_{n} q\right)(x)=\bigoplus_{\sigma \in \mathfrak{S}_{n}} \bigodot_{i=1}^{n}\left(x \oplus r_{i} \oplus s_{\sigma(i)}\right) .
$$

Proof. The product $(p q)(x)$ is a maxpolynomial of degree $m+n$ which is an FCF maxpolynomial by Proposition 2.6. Its set of roots is the union of the roots of $p(x)$ and the roots of $q(x)$ (counting multiplicity). The $k$-th max convolution, i.e., the $k$-th derivative $\left(p \boxplus_{k} q\right)(x)=(p q)^{(k)}(x)$ is again an FCF maxpolynomial by Proposition 2.6] and has degree $m+n-k$. By iterated application of Proposition 2.7 it follows that its roots are the $m+n-k$ maximal roots of $(p q)(x)$.

As for (4.1), it is clear that there exists a permutation $\sigma_{0}$ of $[n]$, such that $r_{1} \oplus s_{\sigma_{0}(1)}, r_{2} \oplus s_{\sigma_{0}(2)}, \ldots, r_{n} \oplus s_{\sigma_{0}(n)}$ are the $n$ maximal numbers among $r_{1}, \ldots, r_{n}, s_{1}, \ldots, s_{n}$. Since each coefficient of $\bigodot_{i=1}^{n}\left(x \oplus r_{i} \oplus\right.$ $\left.s_{\sigma_{0}(i)}\right)$ is greater than or equal to the corresponding coefficient of any other maxpolynomial $\bigodot_{i=1}^{n}\left(x \oplus r_{i} \oplus s_{\sigma(i)}\right)$, the result follows.

The following properties of the max convolution are easily verified.

Proposition 4.3. Let $p(x), p_{1}(x), p_{2}(x), q(x)$ be maxpolynomials. Then

(i) Commutativity: $\left(p \boxplus_{k} q\right)(x)=\left(q \boxplus_{k} p\right)(x)$.

(ii) Distributivity: $\left(\left(p_{1} \oplus p_{2}\right) \boxplus_{k} q\right)(x)=\left(p_{1} \boxplus_{k} q\right)(x) \oplus\left(p_{2} \boxplus_{k} q\right)(x)$.

(iii) Homogeneity: $\left(p_{1} p_{2} \boxplus_{k} q\right)(x)=\left(p_{1} \boxplus_{k} p_{2} q\right)(x)$.

(iv) Leibniz's rule: $\left(p \boxplus_{k} q\right)^{\prime}(x)=\left(p \boxplus_{k+1} q\right)(x)=\left(p^{\prime} \boxplus_{k} q\right)(x) \oplus$ $\left(p \boxplus_{k} q^{\prime}\right)(x)$.

Associativity does not hold in general, but it is satisfied under the following condition, including the case where all maxpolynomials are of degree $n$ and the max convolution is of order $n$.

Proposition 4.4 (Associativity). Let $p_{1}(x), p_{2}(x), p_{3}(x)$ be maxpolynomials of degrees $n_{1}, n_{2}, n_{3} \leq n$, respectively. Then

(i) $\left(\left(p_{1} \boxplus_{n} p_{2}\right) \boxplus_{n} p_{3}\right)(x)=\left(p_{1} \boxplus_{n}\left(p_{2} \boxplus_{n} p_{3}\right)\right)(x)$.

(ii) If $p_{1}(x), p_{2}(x), p_{3}(x)$ are FCF maxpolynomials then the roots of $\left(p_{1} \boxplus_{n} p_{2} \boxplus_{n} p_{3}\right)(x)$ are the maximal $n_{1}+n_{2}+n_{3}-2 n$ roots (including multiplicities) among the roots of $p_{1}(x), p_{2}(x), p_{3}(x)$.

Proof. (i) We distinguish two cases. First assume that $n_{1}+n_{2}+$ $n_{3}<2 n$. Then $\left(\left(p_{1} \boxplus_{n} p_{2}\right) \boxplus_{n} p_{3}\right)(x)=\varepsilon$ for the following reasons: either $n_{1}+n_{2}<n$ and then $\left(p_{1} \boxplus_{n} p_{2}\right)(x)$ is formed by taking the $n$-th derivative of a maxpolynomial of degree $n_{1}+$ $n_{2}<n$, which gives $\varepsilon$, and consequently $\left(\left(p_{1} \boxplus_{n} p_{2}\right) \boxplus_{n} p_{3}\right)(x)=$ 
$\left(\varepsilon \boxplus_{n} p_{3}\right)(x)=\varepsilon$; otherwise $\operatorname{deg}\left(p_{1} \boxplus_{n} p_{2}\right)=n_{1}+n_{2}-n \geq 0$, and then in $\left(\left(p_{1} \boxplus_{n} p_{2}\right) \boxplus_{n} p_{3}\right)(x)$ we take the derivative of order $n$ of a maxpolynomial of degree $\left(n_{1}+n_{2}-n\right)+n_{3}<n$, which again results in $\varepsilon$. Similarly, $\left(p_{1} \boxplus_{n}\left(p_{2} \boxplus_{n} p_{3}\right)\right)(x)=\varepsilon$, and associativity holds.

Thus let us assume that $2 n \leq n_{1}+n_{2}+n_{3} \leq 3 n$ (then necessarily $\left.n_{1}+n_{2} \geq n\right)$. Let $p_{1}(x)=\bigoplus_{i=0}^{n_{1}} a_{i} x^{i}, p_{2}(x)=$ $\bigoplus_{i=0}^{n_{2}} b_{i} x^{i}$ and $p_{3}(x)=\bigoplus_{i=0}^{n_{3}} c_{i} x^{i}$. The coefficients of $\left(p_{1} \boxplus_{n}\right.$ $\left.p_{2}\right)(x)=\left(p_{1} p_{2}\right)^{(n)}(x)$ are the leading $n_{1}+n_{2}+1-n$ coefficients of $\left(p_{1} p_{2}\right)(x)$. That is,

$$
\left(p_{1} \boxplus_{n} p_{2}\right)(x)=\bigoplus_{i=0}^{n_{1}+n_{2}-n} d_{i} x^{i}=\bigoplus_{i=0}^{n_{1}+n_{2}-n}\left(\bigoplus_{\substack{j, k \\ j+k=n+i}} a_{j} b_{k}\right) x^{i}
$$

Then the coefficients of $\left(\left(p_{1} \boxplus_{n} p_{2}\right) \boxplus_{n} p_{3}\right)(x)$ are the $n_{1}+n_{2}+$ $n_{3}+1-2 n$ leading coefficients of $\left(p_{1} p_{2} p_{3}\right)(x)$ :

$$
\begin{aligned}
\left(\left(p_{1} \boxplus_{n} p_{2}\right) \boxplus_{n} p_{3}\right)(x) & =\bigoplus_{i=0}^{n_{1}+n_{2}+n_{3}-2 n}\left(\bigoplus_{\substack{m, l \\
m+l=n+i}} d_{m} c_{l}\right) x^{i} \\
& =\bigoplus_{i=0}^{n_{1}+n_{2}+n_{3}-2 n}\left(\bigoplus_{\substack{m, l \\
m+l=n+i}}\left(\bigoplus_{j+k=n+m} a_{j} b_{k}\right) c_{l}\right) x^{i} \\
& \left.=\bigoplus_{j, k}^{n_{1}+n_{2}+n_{3}-2 n} \bigoplus_{\substack{i=0 \\
j+k+l=2 n+i}} a_{j} b_{k} c_{l}\right) x^{i} .
\end{aligned}
$$

In general, there is an inequality $\leq$ in (4.2). A sharp inequality could be the result of some term $a_{j} b_{k}$ that does not contribute to the computation of $\left(p_{1} \boxplus_{n} p_{2}\right)$ but is part of an expression of the form $a_{j} b_{k} c_{l}$ with $j+k+l=2 n+i$ in (4.2). However, in our case, when $a_{j} b_{k}$ does not contribute to $\left(p_{1} \boxplus_{n} p_{2}\right)$ then $j+k<n$ and hence $j+k+l<n+l \leq n+n_{3} \leq 2 n$. Thus, $a_{j} b_{k} c_{l}$ does not appear in (4.2) either.

By the symmetry of the expression (4.2), the order in which the 3 maxpolynomials are max convolved is irrelevant and associativity follows.

(ii) This is clear by Proposition 4.2 , 
We remark that when $A_{i}, i=0, \ldots, k$, are $n \times n$ matrices over $\mathbb{R}_{\max }$, with full characteristic maxpolynomials $p_{i}(x), i=0, \ldots, k$, then

$$
\left(p_{0} \boxplus_{n} p_{1} \boxplus_{n} \cdots \boxplus_{n} p_{k}\right)(x)=\bigoplus_{\substack{P_{i}, Q_{i} \in \mathcal{P}_{n} \\ i=1, \ldots, k}} \operatorname{perm}\left(x 0 \oplus A_{0} \oplus P_{1} A_{1} Q_{1} \oplus \cdots \oplus P_{k} A_{k} Q_{k}\right),
$$

whose roots are the maximal $n$ roots (including multiplicities) among the roots of $p_{i}(x), i=0, \ldots, k$. This follows from Theorem 4.5 below.

4.2. Max convolution of characteristic maxpolynomials. Let $\mathcal{P}_{n}$ be the group of $n \times n$ max-plus permutation matrices, which are similar to the standard permutation matrices, except that the entries are assigned the values 0 and $\varepsilon$ instead of 1 and 0 , respectively. Given two matrices $A, B \in \mathbb{R}_{\max }^{n \times n}$, we show next that the $n$-th max convolution of their full characteristic maxpolynomials equals the maximum $(\oplus)$, over all permutation matrices $P, Q \in \mathcal{P}_{n}$, of the set of full characteristic maxpolynomials of $A \oplus P B Q$. Recall from (3.2) that by $\eta_{k}(A)$ we denote the maximal value of all minors of order $k$ of a matrix $A$.

Theorem 4.5. Given matrices $A, B$ of order $n$ over $\mathbb{R}_{\max }$, let $p(x)=$ $\bar{\chi}_{A}(x), q(x)=\bar{\chi}_{B}(x)$ be the corresponding full characteristic maxpolynomials and let

$\left(p \boxplus_{n} q\right)(x)=\sum_{k=0}^{n} d_{k} x^{k}$ be their max convolution.

(i) The max convolution can be written as

$$
\left(p \boxplus_{n} q\right)(x)=\bigoplus_{P, Q \in \mathcal{P}_{n}} \bar{\chi}_{A \oplus P B Q}(x) .
$$

(ii) The coefficients of the max convolution are given by

$$
d_{n-k}=\bigoplus_{l=0}^{k} \eta_{l}(A) \eta_{k-l}(B)
$$

(iii) The roots of $\left(p \boxplus_{n} q\right)(x)$ are the maximal $n$ roots (including multiplicities) among the roots of $p(x)$ and $q(x)$.

Proof. Roughly speaking, the proof goes as follows. For each $k$, a maximal minor of order $k$ of $\bigoplus_{P, Q \in \mathcal{P}_{n}} A \oplus P B Q$ is obtained as a maximal (standard) sum of some $0 \leq l \leq k$ elements that lie in $l$ distinct rows and $l$ distinct columns of $A$ and $k-l$ elements of $B$, also in distinct rows and distinct columns, where the positions of the elements of $B$ are independent from the positions of the elements of $A$ since the matrices $P$ and $Q$ allow an arbitrary permutation of the rows and columns of $B$. 
To be precise, let us fix notation first. Let $A=\left(a_{i j}\right), B=\left(b_{i j}\right)$, $p(x)=\sum_{k=0}^{n} a_{k} x^{k}, q(x)=\sum_{k=0}^{n} b_{k} x^{k}$ and $\bigoplus_{P, Q \in \mathcal{P}_{n}} \operatorname{perm}(x 0 \oplus A \oplus$ $P B Q)=\sum_{k=0}^{n} c_{k} x^{k}$. From (3.2) we infer that the coefficients $c_{k}$ are certain maximal minors. To be specific,

$$
\begin{aligned}
c_{n-k} & =\bigoplus_{P, Q} \eta_{k}(A \oplus P B Q) \\
& =\bigoplus_{\sigma, \tau \in \mathfrak{S}_{n}} \bigoplus_{\substack{i_{1}, i_{2}, \ldots, i_{k} \\
j_{1}, j_{2}, \ldots, j_{k}}}\left(a_{i_{1} j_{1}} \oplus b_{\sigma\left(i_{1}\right) \tau\left(j_{1}\right)}\right)\left(a_{i_{2} j_{2}} \oplus b_{\sigma\left(i_{2}\right) \tau\left(j_{2}\right)}\right) \cdots\left(a_{i_{k} j_{k}} \oplus b_{\sigma\left(i_{k}\right) \tau\left(j_{k}\right)}\right),
\end{aligned}
$$

where $\sigma$ and $\tau$ are the inverses of the permutations induced by the respective permutation matrices $P$ and $Q$, and where both tuples $\underline{i}$ and $j$ consist of distinct indices. Using the latter fact, we can expand the products, regroup and relabel the indices to obtain

$$
=\bigoplus_{\sigma, \tau \in \mathfrak{S}_{n}} \bigoplus_{l=0}^{k} \bigoplus_{\substack{i_{1}, i_{2}, \ldots, i_{k} \\ j_{1}, j_{2}, \ldots, j_{k}}} a_{i_{1} j_{1}} a_{i_{2} j_{2}} \cdots a_{i_{l} j_{l}} b_{\sigma\left(i_{l+1}\right) \tau\left(j_{l+1}\right)} b_{\sigma\left(i_{l+2}\right) \tau\left(j_{l+2}\right)} \cdots b_{\sigma\left(i_{k}\right) \tau\left(j_{k}\right)} ;
$$

now $\sigma$ and $\tau$ are arbitrary permutations and after removing duplicated summands we remain with

$$
=\bigoplus_{l=0}^{k} \bigoplus_{\substack{i_{1}, i_{2}, \ldots, i_{l} \\ j_{1}, j_{2}, \ldots, j_{l} \\ i_{1}^{\prime}, i_{2}^{\prime}, \ldots, i_{k-l}^{\prime} \\ j_{1}^{\prime}, j_{2}^{\prime}, \ldots, j_{k-l}^{\prime}}} a_{i_{1} j_{1}} a_{i_{2} j_{2}} \cdots a_{i_{l} j_{l}} b_{i_{1}^{\prime} j_{1}^{\prime}} b_{i_{2}^{\prime} j_{2}^{\prime}} \cdots b_{i_{k-l}^{\prime} j_{k-l}^{\prime}} ;
$$

in this sum the entries of $A$ and $B$ are decoupled and again by (3.2) it is further equal to

$$
\begin{aligned}
& =\bigoplus_{l=0}^{k} \eta_{l}(A) \eta_{k-l}(B) \\
& =\bigoplus_{l=0}^{k} a_{n-l} b_{n-(k-l)} \\
& =\bigoplus_{i+j=2 n-k} a_{i} b_{j},
\end{aligned}
$$

which is indeed $d_{n-k}$, the $(n-k)$ th coefficient of $\left(p \boxplus_{n} q\right)(x)=(p q)^{(n)}(x)$. This completes the proof of (i) and (ii).

So far, we did not make use of the full canonical form. It is, however, essential for item ((iii) and the discussion of the roots of $\left(p \boxplus_{n} q\right)(x)$ First, we observe that both $p(x)$ and $q(x)$ are FCF maxpolynomials by Theorem 3.4. Now, we infer from Proposition 4.1 that the convolution 
$\left(p \boxplus_{n} q\right)(x)$ has the same property and by Proposition 4.2 its roots are the maximal $n$ roots among the roots of $p(x)$ and the roots of $q(x)$.

\subsubsection{Principally dominant matrices.}

Definition 4.6. A square matrix $A \in \mathbb{R}_{\max }^{n \times n}$ is max-plus principally dominant if for every $k \in\{1, \ldots, n\}$ the maximal minor permanent of order $k$ is achieved on a principal submatrix of $A$ of order $k$, see Definition 3.1.

For example, diagonal matrices are principally dominant, as well as diagonally dominant matrices (the diagonal elements are the maximal elements of their rows). Also Gram matrices are principally dominant. In fact, it is easy to generalize Proposition 3.6 to minors and to show that for a Gram matrix $G=A^{T} A$ the maximal minor is

$$
\eta_{k}\left(A^{T} A\right)=\gamma_{1}^{\downarrow} \gamma_{2}^{\downarrow} \cdots \gamma_{k}^{\downarrow}
$$

where the vector $\left(\gamma_{1}^{\downarrow}, \gamma_{2}^{\downarrow}, \ldots, \gamma_{n}^{\downarrow}\right)$ is the nonincreasing rearrangement of the vector $\left(g_{11}, g_{22}, \ldots, g_{n n}\right)=\left(\left\|a_{1}\right\|^{2},\left\|a_{2}\right\|^{2}, \ldots,\left\|a_{n}\right\|^{2}\right)$ of squared norms of the columns of the matrix $A$.

Remark 4.7. A matrix may be symmetric and not principally dominant, e.g. $\left[\begin{array}{ll}0 & 1 \\ 1 & 0\end{array}\right]$, or principally dominant and not symmetric, e.g. $\left[\begin{array}{ll}2 & 1 \\ 0 & 0\end{array}\right]$

Remark 4.8. When $A$ and $B$ are principally dominant then $C=A \oplus B$ is not necessarily principally dominant. For example, in

$$
\left[\begin{array}{llll}
6 & 5 & 0 & 0 \\
5 & 0 & 3 & 0 \\
0 & 2 & 0 & 0 \\
0 & 0 & 0 & 0
\end{array}\right] \oplus\left[\begin{array}{llll}
6 & 5 & 0 & 0 \\
5 & 0 & 0 & 2 \\
0 & 0 & 0 & 0 \\
0 & 3 & 0 & 0
\end{array}\right]=\left[\begin{array}{llll}
6 & 5 & 0 & 0 \\
5 & 0 & 3 & 2 \\
0 & 2 & 0 & 0 \\
0 & 3 & 0 & 0
\end{array}\right]
$$

both $A$ and $B$ are principally dominant, but the maximal minor permanent of order 3 in $C=A \oplus B$, which is $6 \odot 3 \odot 3=12$, is not achieved on any principal submatrix.

The next proposition follows immediately from (3.1) and (3.2).

Proposition 4.9. A matrix $A$ is principally dominant if and only if $\chi_{A}(x)=\bar{\chi}_{A}(x)$.

In general, when $p(x)=\chi_{A}(x)$ and $q(x)=\chi_{B}(x)$ then $\left(p \boxplus_{n} q\right)(x) \leq$ $\bigoplus_{P \in \mathcal{P}_{n}} \chi_{A \oplus P B P^{T}}(x)$. However, when $A$ and $B$ are principally dominant then equality holds and we have the following version of Theorem 4.5, 
which shows that in this case the max convolution can be computed on a set of $n$ ! instead of $(n !)^{2}$ permutation matrices. Recall from (3.1) that by $\delta_{k}(A)$ we denote the maximal value of all principal minors of order $k$ of a matrix $A$.

Theorem 4.10. Given principally dominant matrices $A, B$ of order $n$ over $\mathbb{R}_{\max }$, let $p(x)=\chi_{A}(x), q(x)=\chi_{B}(x)$ be the corresponding characteristic maxpolynomials and let $\left(p \boxplus_{n} q\right)(x)=\sum_{k=0}^{n} d_{k} x^{k}$ be their max convolution.

(i) The max convolution can be written as

$$
\left(p \boxplus_{n} q\right)(x)=\bigoplus_{P \in \mathcal{P}_{n}} \chi_{A \oplus P B P^{T}}(x) .
$$

(ii) The coefficients of the max convolution evaluate to

$$
d_{n-k}=\bigoplus_{l=0}^{k} \delta_{l}(A) \delta_{k-l}(B) .
$$

(iii) The roots of $\left(p \boxplus_{n} q\right)(x)$ are the maximal $n$ roots (including multiplicities) among the roots of $p(x)$ and $q(x)$.

Proof. Since $A, B$ are principally dominant then $p(x)=\chi_{A}(x)=\bar{\chi}_{A}(x)$ and $q(x)=\chi_{B}(x)=\bar{\chi}_{B}(x)$. By Theorem 4.5, we know that $\left(p \boxplus_{n}\right.$ $q)(x)=\bigoplus_{P, Q \in \mathcal{P}_{n}} \bar{\chi}_{A \oplus P B Q}(x)$ and (iii) follows. Moreover, from (4.4) we infer that the coefficients on the left-hand side of (4.6) are

$$
d_{n-k}=\bigoplus_{l=0}^{k} \eta_{l}(A) \eta_{k-l}(B)
$$

and since both $A$ and $B$ are principally dominant, we can replace the minors by principal minors and obtain

$$
=\bigoplus_{l=0}^{k} \delta_{l}(A) \delta_{k-l}(B) .
$$

We have thus proved (ii). To prove(i) it remains to show that the coefficients $c_{k}$ of the maxpolynomial $\bigoplus_{P \in \mathcal{P}_{n}} \chi_{A \oplus P B P^{T}}(x)=\sum_{k=0}^{n} c_{k} x^{k}$ on the right-hand side of (4.6) coincide with those of the maxpolynomial $\bigoplus_{P, Q \in \mathcal{P}_{n}} \bar{\chi}_{A \oplus P B Q}(x)=\sum_{k=0}^{n} d_{k} x^{k}$ which we just computed (4.7).

The idea is as follows. The coefficient $c_{n-k}$ equals the (standard) sum of a maximal permanent of some submatrix $A^{\prime}$ of $A$ of order $l$ and a maximal permanent of a submatrix $B^{\prime}$ of $B$ of order $k-l$, where there is no common row index or common column index between $A^{\prime}$ and the image of $B^{\prime}$ in $P B Q$. But in the principal dominant case, since the maximizing submatrices can be chosen to be principal, the 
permutation matrix $Q$ may be chosen to be equal to $P^{T}$ and thus $A^{\prime}$ and the image of $B^{\prime}$ in $P B P^{T}$ are decoupled.

The details of the calculation are as follows. Applying (3.1) we can write

$$
\begin{aligned}
& c_{n-k}= \bigoplus_{P \in \mathcal{P}_{n}} \delta_{k}\left(A \oplus P B P^{T}\right) \\
&= \bigoplus \bigoplus_{\pi \in \mathfrak{S}_{n}} \bigoplus_{i_{1}, i_{2}, \ldots, i_{k}} \bigoplus_{\sigma \in \mathfrak{S}_{k}}\left(a_{i_{1} i_{\sigma(1)}} \oplus b_{\pi\left(i_{1}\right) \pi\left(i_{\sigma(1)}\right)}\right)\left(a_{i_{2} i_{\sigma(2)}} \oplus b_{\pi\left(i_{2}\right) \pi\left(i_{\sigma(2)}\right)}\right) \\
& \cdots\left(a_{i_{k} i_{\sigma(k)}} \oplus b_{\pi\left(i_{k}\right) \pi\left(i_{\sigma(k)}\right)}\right),
\end{aligned}
$$

where we switched the notation from the permutation matrices $P$ to the corresponding permutations $\pi$. In order to keep the proliferation of indices within manageable bounds, we now replace the sequences of distinct indices by injective functions $g, h:[k] \rightarrow[n]$, where $h=\pi \circ g$, and obtain

$$
\begin{array}{r}
\bigoplus_{g, h:[k] \rightarrow[n]} \bigoplus_{\sigma \in \mathfrak{S}_{k}}\left(a_{g(1) g(\sigma(1))} \oplus b_{h(1) h(\sigma(1)))}\right)\left(a_{g(2) g(\sigma(2))} \oplus b_{h(2) h(\sigma(2)))}\right) \\
\cdots\left(a_{g(k) g(\sigma(k))} \oplus b_{h(k) h(\sigma(k)))}\right) ;
\end{array}
$$

after expanding the product we obtain a sum over all partitions of $[k]$ into two subsets which we denote by index sequences $\underline{i}$ and $\underline{j}$ of size $l$ and $k-l$, respectively:

$$
=\bigoplus_{g, h:[k] \rightarrow[n]} \bigoplus_{l=0}^{k} \bigoplus_{\sigma \in \mathfrak{S}_{k}} a_{g\left(i_{1}\right) g\left(\sigma\left(i_{1}\right)\right)} a_{g\left(i_{2}\right) g\left(\sigma\left(i_{2}\right)\right)} \cdots a_{g\left(i_{l}\right) g\left(\sigma\left(i_{l}\right)\right)} b_{h\left(j_{1}\right) h\left(\sigma\left(j_{1}\right)\right)} b_{h\left(j_{2}\right) h\left(\sigma\left(j_{2}\right)\right)} \cdots b_{h\left(j_{k-l}\right) h\left(\sigma\left(j_{k-l}\right)\right)} ;
$$

now the entries of $A$ and $B$ are decoupled and since both $A$ and $B$ are principally dominant the maximal values are attained when both $\underline{i}$ and $\underline{j}$ are invariant under $\sigma$ and thus give rise to term from a principal minor, yielding

$$
=\bigoplus_{l=0}^{k} \delta_{l}(A) \delta_{k-l}(B)
$$

as claimed.

4.2.2. Symmetric matrices. When the matrix $A$ is symmetric then $\chi_{A}(x)$ and $\bar{\chi}_{A}(x)$ have the same roots (see [16]), that is, $\chi_{A}(x)$ and $\bar{\chi}_{A}(x)$ are functionally equal. However, in general $\chi_{A}(x)$ is not FCF and Theorem 4.10 does not hold as the following example shows. 
Example 4.11. Let $A, B$ be the symmetric matrices

$$
A=\left[\begin{array}{ll}
2 & 0 \\
0 & 0
\end{array}\right], \quad B=\left[\begin{array}{ll}
0 & 1 \\
1 & 0
\end{array}\right] .
$$

Then $\chi_{A}(x)=x^{2} \oplus 2 x \oplus 2$ and its list of roots is $(2,0)$, while $\chi_{B}(x)=$ $x^{2} \oplus x \oplus 2$ with roots $(1,1)$. For each $P \in \mathcal{P}_{2}$ we get

$$
A \oplus P B P^{T}=\left[\begin{array}{ll}
2 & 1 \\
1 & 0
\end{array}\right]
$$

and therefore $\bigoplus_{P \in \mathcal{P}_{2}} \operatorname{perm}\left(x I \oplus A \oplus P B P^{T}\right)=\chi_{A}(x)=x^{2} \oplus 2 x \oplus 2$ with roots $(2,0)$. Thus, in this case we do not get the maximal two eigenvalues among the eigenvalues of $A$ and $B$. Note that for

$$
B^{\prime}=\left[\begin{array}{ll}
1 & 0 \\
0 & 1
\end{array}\right]
$$

we have $\chi_{B^{\prime}}(x)=x^{2} \oplus 1 x \oplus 2$ with the same roots as those of $\chi_{B}$, namely $(1,1)$. However, for each $P \in \mathcal{P}_{2}$,

$$
A \oplus P B^{\prime} P^{T}=\left[\begin{array}{ll}
2 & 0 \\
0 & 1
\end{array}\right]
$$

and therefore $\bigoplus_{P \in \mathcal{P}_{2}} \operatorname{perm}\left(x I \oplus A \oplus P B^{\prime} P^{T}\right)=x^{2} \oplus 2 x \oplus 3$ with roots $(2,1)$. In this case $A$ and $B^{\prime}$ are principally dominant and Theorem 4.10 applies.

Observe also that $\bar{\chi}_{A}(x)=\chi_{A}(x)=x^{2} \oplus 2 x \oplus 2$ with the list of its roots equal to $(2,0)$ and $\bar{\chi}_{B}(x)=x^{2} \oplus 1 x \oplus 2$ with the list of roots $(1,1)$. Moreover, $\bigoplus_{P, Q \in \mathcal{P}_{2}} \operatorname{perm}(x 0 \oplus A \oplus P B Q)=x^{2} \oplus 2 x \oplus 3$ with roots $(2,1)$ and so Theorem 4.5 applies.

4.2.3. Max-row convolution. In 21 the "asymmetric additive convolution" of the characteristic polynomials $p(x)$ and $q(x)$ of $A A^{T}$ and $B B^{T}$, respectively, is defined as

$$
p \boxplus_{n} q(x)=\mathbb{E}_{P, Q} \chi_{(A+P B Q)(A+P B Q)^{T}}(x),
$$

where the expectation is computed by randomly sampling the matrices $P, Q$ over the set of orthonormal matrices equipped with the Haar measure. But if we look at the Gram characteristic polynomial of $(A \oplus$ $P B)^{T}$, i.e., the characteristic polynomial of $\left.\left((A \oplus P B)(A \oplus P B)^{T}\right)\right)^{\circ \frac{1}{2}}$ (note the Hadamard power of $\frac{1}{2}$ ), then the max-plus analogue, the max-row convolution, can be expressed through the already defined max convolution, as shown below.

Theorem 4.12. Let $A, B$ be matrices of order $n$ over $\mathbb{R}_{\max }$, and let $m_{i}$, (resp. $\left.l_{i}\right), i=1, \ldots, n$, be the maximal element of row $i$ in $A$ (resp. B). 
Then the max convolution of the characteristic maxpolynomials $p(x)=$ $\chi_{\widehat{A^{T}}}(x)$ and $q(x)=\chi_{\widehat{B^{T}}}(x)$ is

$$
\left(p \boxplus_{n} q\right)(x)=\bigoplus_{P \in \mathcal{P}_{n}} \chi_{(A \widehat{\oplus P B})^{T}}(x),
$$

and the roots of $\left(p \boxplus_{n} q\right)(x)$ are the maximal $n$ numbers among $\left\{m_{1}, \ldots, m_{n}\right\} \cup$ $\left\{l_{1}, \ldots, l_{n}\right\}$.

Proof. By (3.5), the roots of $\chi_{(A \widehat{\oplus P B})^{T}}(x)$ are the maximal elements of the rows of $A \oplus P B$. Clearly, the maximum in (4.8) is achieved by permuting the rows of $B$ with some $P_{0} \in \mathcal{P}_{n}$ in such a way that the rows of $A \oplus P_{0} B$ contain the maximal numbers among $\left\{m_{1}, \ldots, m_{n}\right\} \cup$ $\left\{l_{1}, \ldots, l_{n}\right\}$.

A max-column convolution can be defined in an analogous way.

\section{HADAMARD PRODUCT}

5.1. Hadamard product of maxpolynomials. Given two maxpolynomials $p(x)=\bigoplus_{i=0}^{n} a_{i} x^{i}, q(x)=\bigoplus_{i=0}^{n} b_{i} x^{i}$ of degree $n$, their max-plus Hadamard product is defined as

$$
(p \circ q)(x)=\bigoplus_{i=0}^{n} a_{i} b_{i} x^{i},
$$

that is, the coefficients of Hadamard product $(p \circ q)(x)$ are the maxproducts (standard sums) of the corresponding coefficients of $p(x)$ and $q(x)$. In general, the roots of $(p \circ q)(x)$ are not the max-products of the corresponding roots of $p(x)$ and $q(x)$. For example, let $p(x)=x^{2} \oplus 4 x \oplus 4$ and let $q(x)=x^{2} \oplus 1 x \oplus 3$. The roots of $p(x)$ are $(4,0)$ and the roots of $q(x)$ are $(1.5,1.5)$, whereas the roots of $(p \circ q)(x)=x^{2} \oplus 5 x \oplus 7$ are $(5,2)$. However, for FCF maxpolynomials we have the following result.

Proposition 5.1. Let $p(x), q(x)$ be FCF maxpolynomials with roots $r_{1} \geq \cdots \geq r_{n}$ and $s_{1} \geq \cdots \geq s_{n}$, respectively. Then $(p \circ q)(x)$ is an FCF maxpolynomial with roots $t_{i}=r_{i} \odot s_{i}=r_{i}+s_{i}, i=1, \ldots, n$. That is,

$$
(p \circ q)(x)=\bigoplus_{\sigma \in \mathfrak{S}_{n}} \bigodot_{i=1}^{n}\left(x \oplus r_{i} \odot s_{\sigma(i)}\right)=\bigodot_{i=1}^{n}\left(x \oplus r_{i} \odot s_{i}\right) .
$$


Proof. Let $p(x)=\bigoplus_{i=0}^{n} a_{i} x^{i}, q(x)=\bigoplus_{i=0}^{n} b_{i} x^{i}$ and let $(p \circ q)(x)=$ $\bigoplus_{i=0}^{n} c_{i} x^{i}$, where $c_{i}=a_{i} b_{i}$, be their Hadamard product. Then

$$
\begin{aligned}
c_{i-1}-c_{i} & =\left(a_{i-1}+b_{i-1}\right)-\left(a_{i}+b_{i}\right)=\left(a_{i-1}-a_{i}\right)+\left(b_{i-1}-b_{i}\right) \\
& \leq\left(a_{i}-a_{i+1}\right)+\left(b_{i}-b_{i+1}\right)=\left(a_{i}+b_{i}\right)-\left(a_{i+1}+b_{i+1}\right) \\
& =c_{i}-c_{i+1}
\end{aligned}
$$

for $i=1, \ldots, n-1$. It follows that $(p \circ q)(x)$ is FCF. Moreover,

$$
t_{i}=c_{i-1}-c_{i}=\left(a_{i-1}-a_{i}\right)+\left(b_{i-1}-b_{i}\right)=r_{i}+s_{i}
$$

for $i=1, \ldots, n$.

As for (5.1), it follows from the fact that the coefficient of $x^{k}$ in each $\bigodot_{i=1}^{n}\left(x \oplus r_{i} \odot s_{\sigma(i)}\right)$ is a max-product of $n-k$ roots $r_{i}$ and $n-k$ roots $s_{j}$, and this term is maximal when $\sigma$ is the identity permutation.

The following properties of the Hadamard product of maxpolynomials are easily verified.

Proposition 5.2. Let $p(x), p_{1}(x), p_{2}(x), q(x), q_{1}(x), q_{2}(x)$ be maxpolynomials. Then

(i) Commutativity: $(p \circ q)(x)=(q \circ p)(x)$.

(ii) Associativity: $\left(\left(p_{1} \circ p_{2}\right) \circ p_{3}\right)(x)=\left(p_{1} \circ\left(p_{2} \circ p_{3}\right)\right)(x)$.

(iii) Distributivity: $\left(\left(p_{1} \oplus p_{2}\right) \circ q\right)(x)=\left(p_{1} \circ q\right)(x) \oplus\left(p_{2} \circ q\right)(x)$.

(iv) $\left(\left(p_{1} \circ q_{1}\right)\left(p_{2} \circ q_{2}\right)\right)(x) \leq\left(\left(p_{1} p_{2}\right) \circ\left(q_{1} q_{2}\right)\right)(x)$.

(v) $\left(\left(p_{1} \circ q_{1}\right) \boxplus_{k}\left(p_{2} \circ q_{2}\right)\right)(x) \leq\left(\left(p_{1} \boxplus_{k} p_{2}\right) \circ\left(q_{1} \boxplus_{k} q_{2}\right)\right)(x)$.

5.2. Hadamard product of matrices. The max-plus Hadamard product of matrices is the analogue of the standard Hadamard product in max-plus algebra. That is, if $A, B$ are two $m \times n$ max-plus matrices then their Hadamard product is $C=A \circ B$, where $C$ is an $m \times n$ matrix satisfying

$$
c_{i j}=a_{i j} \odot b_{i j}
$$

i.e. $c_{i j}=a_{i j}+b_{i j}$ in standard arithmetic. The Hadamard power $A^{\text {ot }}$, $t>0$, of $A=\left(a_{i j}\right)$ is then naturally defined: $\left(A^{\circ t}\right)_{i j}=t a_{i j}$, where the product $t a_{i j}$ is the standard one.

Below we list some properties of the Hadamard product and Hadamard powers. Let $\nu(A)$ denote the largest eigenvalue of $A$, i.e., the largest root of $\chi_{A}(x)$, and let $\|A\|$ denote the largest entry of $A$. As before, we denote by $\widehat{A}$ the Hadamard root of the Gram matrix of $A$, i.e., $\widehat{A}=G^{\circ \frac{1}{2}}=\left(A^{T} A\right)^{\circ \frac{1}{2}}$ and $\widehat{A^{T}}=\left(A A^{T}\right)^{\circ \frac{1}{2}}$. The following properties are known or easy to prove (see, e.g., [27, 28]). 
Proposition 5.3. Let $A, B, A_{1}, \ldots, A_{m} \in \mathbb{R}_{\max }^{n \times n}$ and $t>0$. Then we have

$$
\begin{gathered}
\nu(A \circ B) \leq \nu(A) \nu(B),\|A \circ B\| \leq\|A\|\|B\|, \\
\nu\left(A^{\circ t}\right)=\nu(A)^{t},\left\|A^{\circ t}\right\|=\|A\|^{t}, \\
A_{1}^{\circ t} \cdots A_{m}^{\circ t}=\left(A_{1} \cdots A_{m}\right)^{\circ t}, \\
\nu(A B)=\nu(B A), \\
\|\widehat{A}\|=\left\|\widehat{A^{T}}\right\|=\|A\|=\nu(\widehat{A})=\nu\left(\widehat{A^{T}}\right), \\
\nu\left(A_{1} \circ \cdots \circ A_{m}\right) \leq \nu\left(A_{1} \cdots A_{m}\right) .
\end{gathered}
$$

Similarly to [14, we can prove the following max-plus version of [14, Corollary 3.5, Theorem 3.9, Corollary 3.10]. In the proof it is useful to switch to the isomorphic max-times algebra setting by using the equality $\nu(A)=\log \mu(B)$, where $B$ denotes a non-negative $n \times n$ matrix $B=\left[e^{a_{i j}}\right]$ and $\mu(B)$ denotes the largest max-times eigenvalue of $B$. Then the result follows by replacing the standard product of matrices by the max-times product and by applying the max-times Gelfand formula for $\mu(B)$ (see, e.g., [27, 28, 25, Equality (4)]) in the proofs of [14, Corollary 3.5, Theorem 3.9, Corollary 3.10]. To avoid too much repetition of ideas from [14] we omit the details of the proof.

Theorem 5.4. Let $A, B, A_{1}, \ldots, A_{m} \in \mathbb{R}_{\max }^{n \times n}$. Then we have

$$
\begin{gathered}
\|A \circ B\| \leq \nu\left(A^{T} B\right), \\
\left\|A_{1} \circ A_{2} \circ \cdots \circ A_{m}\right\| \leq \nu\left(\widehat{A_{1}} \circ \widehat{A_{2}} \circ \cdots \circ \widehat{A_{m}}\right) \leq \nu\left(\widehat{A_{1}} \widehat{A_{2}} \cdots \widehat{A_{m}}\right), \\
\left\|A_{1} \circ A_{2} \circ \cdots \circ A_{m}\right\| \leq \nu\left(\widehat{A_{1}^{T}} \circ \widehat{A_{2}^{T}} \circ \cdots \circ \widehat{A_{m}^{T}}\right) \leq \nu\left(\widehat{A_{1}^{T}} \widehat{A_{2}^{T}} \cdots \widehat{A_{m}^{T}}\right) .
\end{gathered}
$$

If $m$ is even then

$$
\begin{aligned}
\left\|A_{1} \circ A_{2} \circ \cdots \circ A_{m}\right\|^{2} & \leq \nu\left(A_{1}^{T} A_{2} A_{3}^{T} A_{4} \cdots A_{m-1}^{T} A_{m}\right) \nu\left(A_{1} A_{2}^{T} A_{3} A_{4}^{T} \cdots A_{m-1} A_{m}^{T}\right) \\
& =\nu\left(A_{1}^{T} A_{2} A_{3}^{T} A_{4} \cdots A_{m-1}^{T} A_{m}\right) \nu\left(A_{m} A_{m-1}^{T} \cdots A_{4} A_{3}^{T} A_{2} A_{1}^{T}\right) .
\end{aligned}
$$

If $m$ is odd then

$$
\left\|A_{1} \circ A_{2} \circ \cdots \circ A_{m}\right\|^{2} \leq \nu\left(A_{1} A_{2}^{T} A_{3} A_{4}^{T} \cdots A_{m-2} A_{m-1}^{T} A_{m} A_{1}^{T} A_{2} A_{3}^{T} A_{4} \cdots A_{m-2}^{T} A_{m-1} A_{m}^{T}\right)
$$




\subsection{Hadamard product of characteristic maxpolynomials.}

Theorem 5.5. Given matrices $A, B$ of order $n$ over $\mathbb{R}_{\max }$, let $p(x)=$ $\bar{\chi}_{A}(x), q(x)=\bar{\chi}_{B}(x)$ be the corresponding full characteristic maxpolynomials and let $(p \circ q)(x)=\sum_{k=0}^{n} d_{k} x^{k}$ be the Hadamard product of the maxpolynomials.

(i) The Hadamard product can be written as

$$
(p \circ q)(x)=\bigoplus_{P, Q \in \mathcal{P}_{n}} \bar{\chi}_{A \circ P B Q}(x) .
$$

(ii) The ordered vector of the roots of $(p \circ q)(x)$ is the Hadamard product of the ordered vectors of the roots of $p(x)$ and $q(x)$.

Proof. The proof is similar to the one of Theorem 4.5, in fact even simpler. When computing the coefficients of $(p \circ q)(x)$ then instead of computing permanent minors of a maximum of submatrices they are computed on (standard) sums of submatrices. Thus, each coefficient $c_{n-k}$ of $\bigoplus_{P, Q \in \mathcal{P}_{n}} \bar{\chi}_{A \circ P B Q}(x)=\sum_{k=0}^{n} c_{k} x^{k}$ is obtained as the (standard) sum of a maximal permanent minor of order $k$ of $A$ and a maximal permanent minor of order $k$ of $B$, where the permutation matrices $P$ and $Q$ make sure that the positions of the elements of $B$ that contribute to the maximal permanent are mapped to the exact positions of the elements of $A$ that contribute to the maximal permanent minor.

To be precise, let $p(x)=\sum_{k=0}^{n} a_{k} x^{k}$ and $q(x)=\sum_{k=0}^{n} b_{k} x^{k}$. By (3.2) each coefficient $c_{n-k}$ is

$$
\begin{aligned}
& c_{n-k}=\bigoplus_{P, Q \in \mathcal{P}_{n}} \eta_{k}(A \circ P B Q) \\
& =\bigoplus_{\sigma, \tau \in \mathfrak{S}_{n} n} \bigoplus_{\substack{i_{1}, i_{2}, \ldots, i_{k} \\
j_{1}, j_{2}, \ldots, j_{k}}} a_{i_{1} j_{1}} b_{\sigma\left(i_{1}\right) \tau\left(j_{1}\right)} a_{i_{2} j_{2}} b_{\sigma\left(i_{2}\right) \tau\left(j_{2}\right)} \cdots a_{i_{k} j_{k}} b_{\sigma\left(i_{k}\right) \tau\left(j_{k}\right)} \\
& =\bigoplus_{\substack{i_{1}, i_{2}, \ldots, i_{k} \\
i_{1}^{\prime}, i_{2}^{\prime}, \ldots, i_{k}^{\prime} \\
j_{1}, j_{2}, \ldots, j_{k} \\
j_{1}^{\prime}, j_{2}^{\prime}, \ldots, j_{k}^{\prime}}} a_{i_{1} j_{1}} a_{i_{2} j_{2}} \cdots a_{i_{k} j_{k}} b_{i_{1}^{\prime} j_{1}^{\prime}} b_{i_{2}^{\prime} j_{2}^{\prime}} \cdots b_{i_{k}^{\prime} j_{k}^{\prime}} \\
& =\eta_{k}(A) \eta_{k}(B) \\
& =a_{n-k} b_{n-k} \text {, }
\end{aligned}
$$

which is $d_{n-k}$, the $(n-k)$ th coefficient of $(p \circ q)(x)$.

Property (ii) follows from Proposition 5.1.

5.4. Hadamard product of characteristic maxpolynomials via multiplicative convolution. When trying to form the analogue of 
Theorem 4.5 with matrix multiplication instead of summation (max), that is, using an expression of the form $\bigoplus_{P, Q \in \mathcal{P}_{n}} \bar{\chi}_{A P B Q}(x)$, we realize that it cannot be done in general and that we have to restrict ourselves to specific classes of matrices. The problem lies in the fact that when performing matrix multiplication we perform a series of scalar products of row vectors by column vectors, and these operations depend on the order of the elements in each vector. Specifically, the scalar product is maximal only when the maximal element in each of the vectors is in the same position.

Hence, it is desired that the matrices $A$ and $B$ match with regard to the positions of the maximal elements in the rows of $A$ and the columns of $B$. For example, $A$ and $B$ match when the maximal elements of the rows of $A$ lie in different columns and the maximal elements of the columns of $B$ lie in different rows (in case there are more than one maximal element in a row of $A$ (resp. a column of $B$ ), each of these elements is a legitimate choice). More generally, it is necessary that the matrices $A^{T}$ and $B$ have the same max-column partition, which is defined as follows. Given a matrix $M \in \mathbb{R}_{\max }^{n \times n}$, for each $j$, $j=1, \ldots, n$, let $m_{j}$ be the $j$-th column of $M$ and let $m_{i_{j} j}=\left\|m_{j}\right\|$ be the maximal element of this column (if the maximum is attained in more than one place then we have more than max-column partition associated with $M$ ). Let $\sigma \in \mathfrak{S}_{n}$ be a permutation which arranges the maximal elements of the columns in ascending order:

$$
m_{i_{\sigma(1)} \sigma(1)} \leq m_{i_{\sigma(2)} \sigma(2)} \leq \cdots \leq m_{i_{\sigma(n)} \sigma(n)} .
$$

Then a max-column partition of $M$ is a partition of $[n]$ into blocks such that $j$ and $k$ lie in the same block if the corresponding matrix elements according to the above order lie in the same row:

$$
j \sim k \Longleftrightarrow i_{\sigma(j)}=i_{\sigma(k)} .
$$

For example, at the bottom of the lattice of partitions is the one where the maximal elements of the columns of $M$ lie in distinct rows: $i_{j} \neq i_{k}$ for each $i \neq k$. The max-column partition of $M$ consists then of $n$ blocks, where each block is a singleton. On the other hand, the top partition is the one where there is a single block with $n$ elements, corresponding to the case where the maximal elements of the columns belong to the same row: $i_{1}=i_{2}=\cdots=i_{n}$.

Given two matrices $A, B \in \mathbb{R}_{\max }^{n \times n}$, such that $A^{T}$ and $B$ share a maxcolumn partition, we show now that the Hadamard product of the Gram characteristic maxpolynomials of $A^{T}$ and $B$ equals the maximum $(\oplus)$, over all permutation matrices $P \in \mathcal{P}_{n}$, of the set of full characteristic maxpolynomials of $A P B$. This maximum is achieved on 
a specific permutation matrix $P_{0}$ which "orients" $B$ towards $A$ by rearranging the rows of $B$. In addition, when we allow multiplication on the right of $B$ with permutation matrices $Q \in \mathcal{P}_{n}$ then we can restrict ourselves to the set of characteristic maxpolynomials instead of full characteristic maxpolynomials. Here the orientation of $B$ towards $A$ is achieved through two specific permutation matrices $P_{0}$ and $Q_{0}$, which rearrange the rows as well as the columns of $B$.

Theorem 5.6. Let $A, B \in \mathbb{R}_{\max }^{n \times n}$ be two matrices, such that $A^{T}$ and $B$ share a max-column partition. Let $p(x)=\chi_{\widehat{A^{T}}}(x), q(x)=\chi_{\widehat{B}}(x)$ be the Gram characteristic maxpolynomials of $A^{T}, B$, respectively. Then

$$
(p \circ q)(x)=\bigoplus_{P \in \mathcal{P}_{n}} \bar{\chi}_{A P B}(x)=\bigoplus_{P, Q \in \mathcal{P}_{n}} \chi_{A P B Q}(x) .
$$

Moreover, there exist permutation matrices $P_{0}, Q_{0} \in \mathcal{P}_{n}$ such that

$$
(p \circ q)(x)=\bar{\chi}_{A P_{0} B}(x)=\chi_{A P_{0} B Q_{0}}(x) .
$$

In addition, the vector of ordered roots of $(p \circ q)(x)$ equals the Hadamard product of the vector of ordered roots of $p(x)$ and the vector of ordered roots of $q(x)$.

Proof. We start with the polynomial

$$
\bigoplus_{k=0}^{n} c_{k} x^{k}:=\bigoplus_{P \in \mathcal{P}_{n}} \bar{\chi}_{A P B}(x)
$$

By (3.2) the coefficients are given by

$$
\begin{aligned}
c_{n-k} & =\bigoplus_{P \in \mathcal{P}_{n}} \eta_{k}(A P B) \\
& =\bigoplus_{\pi \in \mathfrak{S}_{n}} \bigoplus_{\substack{i_{1}, i_{2}, \ldots, i_{k} \\
j_{1}, j_{2}, \ldots, j_{k}}} \bigoplus_{l_{1}, l_{2}, \ldots, l_{k}} a_{i_{1} l_{1}} b_{\pi\left(l_{1}\right) j_{1}} a_{i_{2} l_{2}} b_{\pi\left(l_{2}\right) j_{2}} \cdots a_{i_{k} l_{k}} b_{\pi\left(l_{k}\right) j_{k}} ;
\end{aligned}
$$

each summation can be estimated with the Cauchy-Bunyakovsky-Schwarz inequality (3.4)

$$
\leq \bigoplus_{\substack{i_{1}, i_{2}, \ldots, i_{k} \\ j_{1}, j_{2}, \ldots, j_{k}}}\left\|a_{i_{1}}\right\|\left\|b_{j_{1}}\right\|\left\|a_{i_{2}}\right\|\left\|b_{j_{2}}\right\| \cdots\left\|a_{i_{k}}\right\|\left\|b_{j_{k}}\right\|,
$$

where by $a_{i}$ we denote the rows of $A$ and by $b_{j}$ the columns of $B$. Now by (4.5) this maximum is

$$
=\eta_{k}\left(\widehat{A^{T}}\right) \eta_{k}(\widehat{B})
$$

and we have a chain of equalities interrupted by one inequality. In order for equality to hold in this inequality, equality must hold in each 
Cauchy-Bunyakovsky-Schwarz inequality, which boils down to the requirement that $A^{T}$ and $B$ share a max-column partition.

Moreover, there exists a permutation matrix $P_{0}$ such that

$$
(p \circ q)(x)=\bigoplus_{P \in \mathcal{P}_{n}} \bar{\chi}_{A P B}(x)=\bar{\chi}_{A P_{0} B}(x) .
$$

Indeed, the permutation matrix $P_{0}$ should arrange the rows of $B$ to match the positions of the maximal elements in the rows of $A$. Let $r_{1} \geq r_{2} \geq \cdots \geq r_{n}$ be the maximal elements of the rows of $A$ (the columns of $A^{T}$ ), that is, the roots of $\chi_{\widehat{A^{T}}}(x)$, the Gram characteristic maxpolynomial of $A^{T}$, and let $s_{1} \geq s_{2} \geq \cdots \geq s_{n}$ be the maximal elements of the columns of $B$. Since $A^{T}$ and $B$ share a max-column partition, there exists a permutation matrix $P_{0}$ such that if $r_{i}$ is in column $k_{i}$ then $s_{i}$ is in row $k_{i}$ of $P_{0} B$, for $i=1, \ldots, n$.

The elements $t_{j}=r_{j}+s_{j}$ lie in $n$ different rows and $n$ different columns of $A P_{0} B$. By multiplying on the right with the appropriate permutation matrix $Q_{0}$, these elements can be moved to the diagonal and it follows that $\bar{\chi}_{A P_{0} B}(x)=\chi_{A P_{0} B Q_{0}}(x)$.

By Theorem 3.4 and Proposition 5.1 the set of roots of $(p \circ q)(x)$ is the Hadamard product of the roots of $p(x)$ and the roots of $q(x)$. In fact, it is easily verified that $p(x)$ and $q(x)$ are FCF maxpolynomials without the need for Theorem 3.4 since one can treat $\widehat{A^{T}}$ and $\widehat{B}$ as diagonal matrices, as the elements $t_{j}$ are the only elements that contribute to the characteristic maxpolynomials $p(x)$ and $q(x)$.

Remark 5.7. It is clear that the condition in Theorem 5.6 about $A^{T}$ and $B$ sharing a max-column partition is not only sufficient but also necessary for equality (5.3) to hold.

Example 5.8. Let $A, B$ be the matrices

$$
A=\left[\begin{array}{rrrr}
2 & 0 & 3 & -1 \\
0 & 0 & 1 & 1 \\
-2 & 2 & 2 & 1 \\
2 & -1 & 1 & 1
\end{array}\right], \quad B=\left[\begin{array}{rrrr}
0 & 0 & -2 & 2 \\
-2 & 1 & -1 & -1 \\
-1 & 0 & -3 & -1 \\
-1 & -2 & -1 & 0
\end{array}\right]
$$

The Gram characteristic maxpolynomial of $A^{T}$ is the characteristic maxpolynomial of

$$
\widehat{A^{T}}=\left(A A^{T}\right)^{\circ \frac{1}{2}}=\left[\begin{array}{cccc}
3 & 2 & \frac{5}{2} & 2 \\
2 & 1 & \frac{3}{2} & 1 \\
\frac{5}{2} & \frac{3}{2} & 2 & \frac{3}{2} \\
2 & 1 & \frac{3}{2} & 2
\end{array}\right]
$$


which is

$$
\begin{aligned}
p(x) & =x^{4} \oplus 3 x^{3} \oplus 5 x^{2} \oplus 7 x \oplus 8 \\
& =(x \oplus 3)(x \oplus 2)^{2}(x \oplus 1) .
\end{aligned}
$$

Then

$$
\widehat{B}=\left(B^{T} B\right)^{\circ \frac{1}{2}}=\left[\begin{array}{rrrr}
0 & 0 & -1 & 1 \\
0 & 1 & 0 & 1 \\
-1 & 0 & -1 & 0 \\
1 & 1 & 0 & 2
\end{array}\right]
$$

whose characteristic maxpolynomial is

$$
\begin{aligned}
q(x) & =x^{4} \oplus 2 x^{3} \oplus 3 x^{2} \oplus 3 x \oplus 2 \\
& =(x \oplus 2)(x \oplus 1)(x \oplus 0)(x \oplus-1) .
\end{aligned}
$$

The Hadamard product of $p(x)$ and $q(x)$ is

$$
\begin{aligned}
(p \circ q)(x) & =(0 \odot 0) x^{4} \oplus(3 \odot 2) x^{3} \oplus(5 \odot 3) x^{2} \oplus(7 \odot 3) x \oplus(8 \odot 2) \\
& =x^{4} \oplus 5 x^{3} \oplus 8 x^{2} \oplus 10 x \oplus 10 \\
& =(x \oplus 5)(x \oplus 3)(x \oplus 2)(x \oplus 0) .
\end{aligned}
$$

We see that the roots of $(p \circ q)(x)$ are

$$
(5,3,2,0)=(3,2,2,1) \circ(2,1,0,-1),
$$

the Hadamard product of the ordered roots of $p(x)$ and $q(x)$.

Let us now look at the maximal elements of the rows of $A$ and columns of $B$ (marked with an asterisk):

$$
A=\left[\begin{array}{rrrr}
2 & 0 & 3^{\star} & -1 \\
0 & 0 & 1^{\star} & 1^{\star} \\
-2 & 2^{\star} & 2^{\star} & 1 \\
2^{\star} & -1 & 1 & 1
\end{array}\right], \quad B=\left[\begin{array}{rrrr}
0^{\star} & 0 & -2 & 2^{\star} \\
-2 & 1^{\star} & -1^{\star} & -1 \\
-1 & 0 & -3 & -1 \\
-1 & -2 & -1^{\star} & 0
\end{array}\right] .
$$

The ordered list of column-maximal elements in $B$ is $(-1,0,1,2)$, referring to columns $(3,1,2,4)$. The corresponding list of rows of these elements is $((2,4), 1,2,1)$, where the pair $(2,4)$ refers to the maximal element of the third column, namely

-1 , which occurs in row 2 and in row 4 . We see that the matrix $B$ admits two max-column partitions. If we choose the second row in the third column then the partition is $\{(1,3),(2,4)\}$ : the first and third ordered elements ( -1 and 1$)$ lie in the same row (second row), whereas the second and fourth elements (0 and 2$)$ lie also in the same row (first row). The second partition is $\{(1),(3),(2,4))\}$, which is obtained by choosing the 4-th row as the position of the maximal element of the third column. 
The matrix $A$ admits several max-row partitions (max-column partitions of $A^{T}$ ), including the partition $\left.\{(1),(3),(2,4))\right\}$, which is also a max-column partition of $B$. The chosen maximal elements in the rows of $A$ (in ascending order) are $a_{24}, a_{33}, a_{41}, a_{13}$, and the chosen maximal elements in the columns of $B$ are $b_{43}, b_{11}, b_{22}, b_{14}$ :

$$
A=\left[\begin{array}{rrrr}
2 & 0 & 3^{\star} & -1 \\
0 & 0 & 1 & 1^{\star} \\
-2 & 2 & 2^{\star} & 1 \\
2^{\star} & -1 & 1 & 1
\end{array}\right], \quad B=\left[\begin{array}{rrrr}
0^{\star} & 0 & -2 & 2^{\star} \\
-2 & 1^{\star} & -1 & -1 \\
-1 & 0 & -3 & -1 \\
-1 & -2 & -1^{\star} & 0
\end{array}\right] .
$$

The list of rows of $A$ ordered by their maximal elements (in ascending order) is $(2,3,4,1)$, with corresponding columns $(4,3,1,3)$. The list of columns of $B$ ordered by their maximal element is $(3,1,2,4)$ with corresponding rows $(4,1,2,1)$. In order to match the positions of the chosen maximal elements of the rows of $A$ and the columns of $B$ we need to transfer $(4,1,2,1)$ to $(4,3,1,3)$, that is, perform the moves $1 \rightarrow 3$ and $2 \rightarrow 1$. Hence we need to move the first row of $B$ to the third and to move the second row of $B$ to the first. This can be achieved via the permutation matrix $P_{0}$ that corresponds to the permutation (213):

$$
P_{0} B=\left[\begin{array}{llll}
\varepsilon & 0 & \varepsilon & \varepsilon \\
\varepsilon & \varepsilon & 0 & \varepsilon \\
0 & \varepsilon & \varepsilon & \varepsilon \\
\varepsilon & \varepsilon & \varepsilon & 0
\end{array}\right]\left[\begin{array}{rrrr}
0^{\star} & 0 & -2 & 2^{\star} \\
-2 & 1^{\star} & -1 & -1 \\
-1 & 0 & -3 & -1 \\
-1 & -2 & -1^{\star} & 0
\end{array}\right]=\left[\begin{array}{rrrr}
-2 & 1^{\star} & -1 & -1 \\
-1 & 0 & -3 & -1 \\
0^{\star} & 0 & -2 & 2^{\star} \\
-1 & -2 & -1^{\star} & 0
\end{array}\right]
$$

Then, multiplying $A$ with $P_{0} B$ gives

$$
A P_{0} B=\left[\begin{array}{rrrr}
2 & 0 & 3^{\star} & -1 \\
0 & 0 & 1 & 1^{\star} \\
-2 & 2 & 2^{\star} & 1 \\
2^{\star} & -1 & 1 & 1
\end{array}\right]\left[\begin{array}{rrrr}
-2 & 1^{\star} & -1 & -1 \\
-1 & 0 & -3 & -1 \\
0^{\star} & 0 & -2 & 2^{\star} \\
-1 & -2 & -1^{\star} & 0
\end{array}\right]=\left[\begin{array}{rrrr}
3 & 3 & 1 & 5^{\star} \\
1 & 1 & 0^{\star} & 3 \\
2^{\star} & 2 & 0 & 4 \\
1 & 3^{\star} & 1 & 3
\end{array}\right] \text {. }
$$

The marked elements in $A P_{0} B$ are the roots of the full characteristic maxpolynomial: $\bar{\chi}_{A P_{0} B}(x)=(x \oplus 5)(x \oplus 3)(x \oplus 2)(x \oplus 0)=(p \circ q)(x)$.

Finally, if we want the roots of the full characteristic maxpolynomial to lie on the diagonal (and then the characteristic maxpolynomial equals the full characteristic maxpolynomial), then we need to permute the columns of $A P_{0} B$ by multiplying on the right with the matrix $Q_{0}$, which represents the permutation (1324):

$$
A P_{0} B Q_{0}=\left[\begin{array}{rrrr}
3 & 3 & 1 & 5^{\star} \\
1 & 1 & 0^{\star} & 3 \\
2^{\star} & 2 & 0 & 4 \\
1 & 3^{\star} & 1 & 3
\end{array}\right]\left[\begin{array}{llll}
\varepsilon & \varepsilon & 0 & \varepsilon \\
\varepsilon & \varepsilon & \varepsilon & 0 \\
\varepsilon & 0 & \varepsilon & \varepsilon \\
0 & \varepsilon & \varepsilon & \varepsilon
\end{array}\right]=\left[\begin{array}{rrrr}
5^{\star} & 1 & 3 & 3 \\
3 & 0^{\star} & 1 & 1 \\
4 & 0 & 2^{\star} & 2 \\
3 & 1 & 1 & 3^{\star}
\end{array}\right]
$$


Clearly, $\chi_{A P_{0} B Q_{0}}(x)=\bar{\chi}_{A P_{0} B}(x)$.

Acknowledgement. We thank Bettina Klinz for interesting and fruitful discussions.

\section{REFERENCES}

[1] Marianne Akian, Ravindra B. Bapat, and Stéphane Gaubert, Generic asymptotics of eigenvalues using Min-Plus algebra, In Proceedings of the Workshop on Max-Plus Algebras, IFAC SSSC'01, Elsevier, 2001.

[2] François L. Baccelli, Guy Cohen, Geert J. Olsder, and Jean-Pierre Quadrat, Synchronization and linearity, Wiley Series in Probability and Mathematical Statistics: Probability and Mathematical Statistics, John Wiley \& Sons, Ltd., Chichester, 1992, An algebra for discrete event systems.

[3] Ravindra B. Bapat, A max version of the Perron-Frobenius theorem, Linear Algebra Appl. 275/276 (1998), 3-18.

[4] Dimitris Bertsimas and John M. Tsitsiklis, Introduction to linear optimization, Athena Scientific, Belmont, Massachusetts, 1997.

[5] Rainer Burkard, Mauro Dell'Amico, and Silvano Martello, Assignment problems (revised reprint), Society for Industrial and Applied Mathematics (SIAM), 2012.

[6] Peter Butkovič, Max-linear systems: theory and algorithms, Springer Monographs in Mathematics, Springer-Verlag London, Ltd., London, 2010.

[7] Peter Butkovič, R. A. Cuninghame-Green, and Stephane Gaubert, Reducible spectral theory with applications to the robustness of matrices in max-algebra, SIAM J. Matrix Anal. Appl. 31 (2009), no. 3, 1412-1431.

[8] Ray A. Cuninghame-Green, The characteristic maxpolynomial of a matrix, J. Math. Anal. Appl. 95 (1983), no. 1, 110-116.

[9] Ray A. Cuninghame-Green and P. F. J. Meijer, An algebra for piecewise-linear minimax problems, Discrete Appl. Math. 2 (1980), no. 4, 267-294.

[10] George B. Dantzig and Mukund N. Thapa, Linear programming. 1, Springer Series in Operations Research, Springer-Verlag, New York, 1997.

[11] Bart De Schutter and Bart De Moor, The QR decomposition and the singular value decomposition in the symmetrized max-plus algebra revisited, SIAM Rev. 44 (2002), no. 3, 417-454, Reprint of SIAM J. Matrix Anal. App. 19 (1998), no. 2, 378-406 (electronic).

[12] Mauro Dell'Amico and Silvano Martello, The $k$-cardinality assignment problem, Discrete Appl. Math. 76 (1997), no. 1-3, 103-121.

[13] Jean Dieudonné, La théorie analytique des polynômes d'une variable (à coefficients quelconques), Mémorial des sciences mathématiques, fascicule 93, Gauthier-Villars, Paris, 1938.

[14] Roman Drnovšek and Aljoša Peperko, Inequalities on the spectral radius and the operator norm of Hadamard products of positive operators on sequence spaces, Banach J. Math. Anal. 10 (2016), no. 4, 800-814.

[15] Nathan Grigg and Nathan Manwaring, An Elementary Proof of the Fundamental Theorem of Tropical Algebra, ArXiv e-prints (2007), arXiv:0707.2591.

[16] James Hook, Max-plus singular values, Linear Algebra Appl. 486 (2015), 419442. 
[17] Zur Izhakian, Manfred Knebusch, and Louis Rowen, Algebraic structures of tropical mathematics, Tropical and idempotent mathematics and applications, Contemp. Math., vol. 616, Amer. Math. Soc., Providence, RI, 2014, pp. 125150.

[18] Grigory L. Litvinov, The Maslov dequantization, idempotent and tropical mathematics: a brief introduction, J. Math. Sci. 140 (2007), 426-444, Translated from Zap. Nauchn. Sem. (POMI), 326, (2005), 145-182.

[19] Grigory L. Litvinov and Victor P. Maslov (eds.), Idempotent mathematics and mathematical physics, Contemporary Mathematics, vol. 377, American Mathematical Society, Providence, RI, 2005, Papers from the International Workshop held in Vienna, February 3-10, 2003.

[20] Adam W. Marcus, Polynomial convolutions and (finite) free probability, 2016, preprint on webpage at https://web.math.princeton.edu/ amarcus/index.html.

[21] Adam W. Marcus, Daniel A. Spielman, and Nikhil Srivastava, Finite free convolutions of polynomials, 2015.

[22] _ Interlacing families II: Mixed characteristic polynomials and the Kadison-Singer problem, Ann. of Math. (2) 182 (2015), no. 1, 327-350.

[23] Morris Marden, Geometry of polynomials, Second edition. Mathematical Surveys, No. 3, American Mathematical Society, Providence, R.I., 1966.

[24] Jiří Matoušek and Bernd Gärtner, Understanding and using linear programming, Universitext, Springer, 2007.

[25] Vladimir Müller and Aljoša Peperko, On the spectrum in max algebra, Linear Algebra Appl. 485 (2015), 250-266.

[26] Lior Pachter and Bernd Sturmfels (eds.), Algebraic statistics for computational biology, Cambridge University Press, New York, 2005.

[27] Aljoša Peperko, On the max version of the generalized spectral radius theorem, Linear Algebra Appl. 428 (2008), no. 10, 2312-2318.

[28] _ Bounds on the generalized and the joint spectral radius of Hadamard products of bounded sets of positive operators on sequence spaces, Linear Algebra Appl. 437 (2012), no. 1, 189-201.

[29] Qazi I. Rahman and Gerhard Schmeisser, Analytic theory of polynomials, London Mathematical Society Monographs. New Series, vol. 26, The Clarendon Press, Oxford University Press, Oxford, 2002.

[30] Alexander Schrijver, Theory of linear and integer programming, WileyInterscience Series in Discrete Mathematics, John Wiley \& Sons, Ltd., Chichester, 1986, A Wiley-Interscience Publication. MR 874114

[31] Gábor Szegö, Bemerkungen zu einem Satz von J. H. Grace über die Wurzeln algebraischer Gleichungen, Math. Z. 13 (1922), no. 1, 28-55.

[32] Yen-Lung Tsai, Working with tropical meromorphic functions of one variable, Taiwanese J. Math. 16 (2012), no. 2, 691-712. MR 2892907

[33] Joseph L. Walsh, On the location of the roots of certain types of polynomials, Trans. Amer. Math. Soc. 24 (1922), no. 3, 163-180. 
(A. Rosenmann and F. Lehner) Institute of Discrete Mathematics, Graz University of Technology, Steyrergasse 30, A-8010 Graz, Austria

E-mail address, A. Rosenmann: rosenmann@math.tugraz.at

E-mail address, F. Lehner: lehner@math.tu-graz.ac.at

(A. Peperko) Faculty of Mechanical Engineering, University of Ljubljana, AšKerČeva 6, SI-1000 Luubluana, Slovenia; Institute of Mathematics, Physics and Mechanics, Jadranska 19, SI-1000 Luubluana, SloveNIA.

E-mail address, A. Peperko: aljosa.peperko@fmf.uni-lj.si 